\title{
Drosophila Exo70 Is Essential for Neurite Extension and Survival under Thermal Stress
}

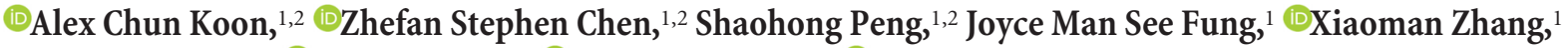 \\ Kayly M. Lembke, ${ }^{6}{ }^{\oplus}$ Hoi Kin Chow, ${ }^{2}{ }^{1}$ C. Andrew Frank, ${ }^{6}{ }^{\circ}$ Liwen Jiang, ${ }^{2,3}$ Kwok-Fai Lau, ${ }^{2,3}$ \\ and $\oplus_{0}$ Ho Yin Edwin Chan ${ }^{1,2,3,4,5}$ \\ ${ }^{1}$ Laboratory of Drosophila Research, ${ }^{2}$ Biochemistry Program, ${ }^{3}$ Cell and Molecular Biology Program, ${ }^{4}$ Molecular Biotechnology Program, ${ }^{5}$ School of Life \\ Sciences, Faculty of Science, Gerald Choa Neuroscience Centre, Chinese University of Hong Kong, Shatin, N.T., Hong Kong SAR, People’s Republic of China, \\ and 'Interdisciplinary Graduate Program in Neuroscience, Department of Anatomy and Cell Biology, Carver College of Medicine, University of Iowa,
}

Iowa City, Iowa 52242

The octomeric exocyst complex governs the final step of exocytosis in both plants and animals. Its roles, however, extend beyond exocytosis and include organelle biogenesis, ciliogenesis, cell migration, and cell growth. Exo70 is a conserved component of the exocyst whose function in Drosophila is unclear. In this study, we characterized two mutant alleles of Drosophila exo70. exo70 mutants exhibit reduced synaptic growth, locomotor activity, glutamate receptor density, and mEPSP amplitude. We found that presynaptic Exo70 is necessary for normal synaptic growth at the neuromuscular junction (NMJ). At the neuromuscular junction, exo70 genetically interacts with the small GTPase ralA to regulate synaptic growth. Loss of Exo70 leads to the blockage of JNK signaling-, activity-, and temperatureinduced synaptic outgrowths. We showed that this phenotype is associated with an impairment of integral membrane protein transport to the cell surface at synaptic terminals. In octopaminergic motor neurons, Exo70 is detected in synaptic varicosities, as well as the regions of membrane extensions in response to activity stimulation. Strikingly, mild thermal stress causes severe neurite outgrowth defects and pharate adult lethality in exo70 mutants. exo70 mutants also display defective locomotor activity in response to starvation stress. These results demonstrated that Exo70 is an important regulator of induced synaptic growth and is crucial for an organism's adaptation to environmental changes.

Key words: Exoc7; exocyst; exocytosis; membrane trafficking; plasticity; synaptic growth

\section{Significance Statement}

The exocyst complex is a conserved protein complex directing secretory vesicles to the site of membrane fusion during exocytosis, which is essential for transporting proteins and membranes to the cell surface. Exo70 is a subunit of the exocyst complex whose roles in neurons remain elusive, and its function in Drosophila is unclear. In Drosophila, Exo70 is expressed in both glutamatergic and octopaminergic neurons, and presynaptic Exo70 regulates synaptic outgrowth. Moreover, exo70 mutants have impaired integral membrane transport to the cell surface at synaptic terminals and block several kinds of induced synaptic growth. Remarkably, elevated temperature causes severe arborization defects and lethality in exo70 mutants, thus underpinning the importance of Exo70 functions in development and adaptation to the environment.

\section{Introduction}

The evolutionarily conserved exocyst complex tethers post-Golgi secretory vesicles to the site of membrane fusion during exocyto- sis (Novick et al., 1980; TerBush et al., 1996), which is essential for delivering integral membrane proteins to the cell surface or releases materials into the extracellular space. Moreover, exocytosis provides a source of phospholipids for membrane extension

Chinese Academy of Science, People's Republic of China), and Stephane Noselli (Institute of Biology, Valrose, France) for sharing of reagents.

The authors declare no competing financial interests.

Correspondence should be addressed to Dr. Ho Yin Edwin Chan, School of Life Sciences, The Chinese University of Hong Kong, Shatin, N.T., Hong Kong SAR, People's Republic of China. E-mail: hyechan@cuhk.edu.hk. DOI:10.1523/JNEUROSCI.0620-18.2018

Copyright $\odot 2018$ the authors $\quad 0270-6474 / 18 / 388071-16 \$ 15.00 / 0$ 
structures, such as filopodia or cilia (Biondini et al., 2016; Lobo et al., 2017). The exocyst complex is widely believed to bridge the SNAREs on opposing membranes, docking the vesicle to the receiving membrane and allowing lipid fusion (Hong and Lev, 2014). Interestingly, despite its wide range of functions and regulation of exocytosis, the exocyst is not involved in the release of synaptic vesicles in neurons (Murthy et al., 2003). It does, however, play a role in the arborization of neurons. Previous studies have shown that the exocyst is enriched in axon growth cones and filopodia, where it is involved in membrane extension by mediating vesicle fusion with the plasma membrane (Hazuka et al., 1999; Dupraz et al., 2009). At the postsynaptic cell, the exocyst facilitates glutamate receptor trafficking and insertion into the postsynaptic membrane (Liebl et al., 2005; Gerges et al., 2006).

Although much is known about the functions of the exocyst complex, the precise roles of individual subunits remain rudimentary. One of the subunits of the exocyst complex is Exo70. In Arabidopsis, among the 23 Exo70 homologs, Exo70A1 was shown to regulate conventional exocytosis and plant cell growth (Hála et al., 2008), whereas Exo70E2 is associated with EXPO (Exocystpositive organelle), a novel double-membrane organelle, which mediates cytosol-to-plasma membrane unconventional protein secretion (Wang et al., 2010; Ding et al., 2014a, b). In mammals, Exo70 (aalso known as Exoc7) is required for the targeting of Glut4 to the plasma membrane by insulin (Inoue et al., 2003). Recent studies on Exo70 have associated it with cell adhesion (Hertzog et al., 2012), cell migration (Zhao et al., 2013; Ma et al., 2016), and invasion (Zuo et al., 2006), with implications to cancer progression (Zhao et al., 2016; Xiao et al., 2017). Compounds that suppress exocytosis by targeting Exo70 are also being studied in the hope of shedding light in cancer drug designs (Zhang et al., 2016). In mammalian neurons, postsynaptic Exo70 mediates AMPA receptor insertion directly into the postsynaptic density (Gerges et al., 2006). Presynaptically, Exo70 is required for the addition of new membrane and axon elongation (Dupraz et al., 2009), which involves the hydrolysis of GTP by TC10 (Fujita et al., 2013).

To clarify a role for the exocyst and its subunit Exo70 in synaptic growth and function, we characterized two mutant alleles of Drosophila exo70. We demonstrated that exo70 mutants displayed locomotor defects and synaptic outgrowth defects at the neuromuscular junction (NMJ). exo70 mutants also exhibited decreased glutamate receptor density and reduced mEPSP amplitude. Presynaptic Exo70 is localized in synaptic boutons, regulating synaptic growth together with the small GTPase RalA. RalA is known to interact with the exocyst, and negatively regulate the JNK signaling pathway (Balakireva et al., 2006). However, we found the Exo70 regulation of synaptic growth to be JNK signaling-independent. Indeed, the exo70 mutations blocked JNK signaling-induced synaptic growth, activity-induced rapid arbor extension, and elevated temperature-induced synaptic growth. Strikingly, rearing exo 70 mutants at $29^{\circ} \mathrm{C}$ resulted in severe neurite outgrowth defects and pharate adult lethality. These experiments demonstrated that Exo70 is not only essential for the organism's development, but also for survival under thermal stress.

\section{Materials and Methods}

Fly stocks. $w^{1118}$ (BDSC catalog \#3605, RRID:BDSC_3605), exo70 ${ }^{K G 08051}$ (14932), exo70 $0^{\text {MBO4553 }}$ (24721), elav-GAL4 (BDSC catalog \#458, RRID: BDSC_458), elav ${ }^{G S}$-GAL4 (BDSC catalog \#43642, RRID:BDSC_43642), Df(3L)BSC815 (BDSC catalog \#27576, RRID:BDSC_27576), Tdc2-GAL4 (BDSC catalog \#9313, RRID:BDSC_9313), ralA ${ }^{E E 1}$ (BDSC catalog
\#25095, RRID:BDSC_25095), UAS-hep ${ }^{\text {CA }}$ (BDSC catalog \#9306, RRID: BDSC_9306), UAS-bsk ${ }^{D N}$ (BDSC catalog \#6409, RRID:BDSC_6409), and UAS-CD8::GFP (BDSC catalog \#5137, RRID:BDSC_5137) were acquired from Bloomington Drosophila Stock Center. C380-GAL4 (also known as BG380) (BDSC catalog \#42736, RRID:BDSC_42736) and C57-GAL4 (also known as BG57) (BDSC catalog \#32556, RRID: BDSC_32556) (Budnik et al., 1996) were obtained from Vivian Budnik laboratory (University of Massachusetts). $\mathrm{ralA}^{P G 89}$ was obtained from Thomas Schwarz laboratory (Harvard Medical School). exo $70^{G D 12140}$ $d s R N A$ (27867) and exo70 KK101154 $-d s R N A$ (103717) were acquired from Vienna Drosophila Resource Center.

Drosophila rearing conditions and gender selection. Flies were reared in standard Drosophila medium at $25^{\circ} \mathrm{C}$, except when otherwise indicated. Animals used in RNAi experiments were reared at $29^{\circ} \mathrm{C}$ to increase the knockdown efficiency. Animals overexpressing UAS-hep ${ }^{C A}$ in motor neurons were reared at $21.5^{\circ} \mathrm{C}$ to reduce lethality. Both male and female larvae/flies were used in all experiments, with the exception of experiments involving $r a l A^{P G 89}, r a l A^{E E 1}$, and $U A S-b s k^{D N}$, in which only female animals were used. This is because the ralA gene and the UAS-bsk $k^{D N}$ insert are both located on the X chromosome.

Generation of Drosophila Exo70 antibodies. Exo70 antibodies were raised against the first 400 amino acids of Drosophila Exo70 via the Genscript's PolyExpress Premium package. In brief, rabbit sera from at least 10 animals were prescreened using immunohistochemistry at the Drosophila larval NMJ fixed in either 4\% PFA or Bouin's fixative. Two rabbits (named Maguro and Sake) of the lowest background were selected for immunization. The collected bleeds were affinity purified independently, resulting in two independent Exo70 antibodies (anti-Exo70 Maguro and anti-Exo70 ${ }^{\text {Sake }}$.

Generation of UAS-exo70. Drosophila exo70 was cloned from an exo70 cDNA in pcDNA3.1+/C- $(K) D Y K$ vector purchased from Genscript (clone ID OFa20731). The forward primer used was 5'-GCG AAT TCA TGA ACA ATT TGG ACA GTT CAT TG-3' ${ }^{\prime}$, with an EcoRI restriction site introduced to the $5^{\prime}$ end of the primer. The reverse primer used was 5'-GCG CGG CCG CTT ATC ACT TAT CGT CGT CAT CCT TG-3', with a NotI restriction site introduced to the $5^{\prime}$ end of the primer. These primers were used to amplify the exo $70 \mathrm{cDNA}$ together with a DYK tag and a stop codon in the vector. The PCR fragment was then subcloned into $P U A S T$ vector, and subsequently sequenced. The $p U A S T$-exo70$D Y K$ plasmid was sent to BestGene for microinjection, and 10 transformants were obtained. Because the exo70 gene is located on the third chromosome, we chose transformants with the UAS-exo70 transgene inserted on the second chromosome for further characterization. UASexo70 transformant \#1-M5 was used in this study due to its robust transgene expression, and without detectable exo70 transgene expression when GAL4 is absent.

Verification of recombination. Because both the exo70 gene and C57 muscle driver are on the third chromosome, we performed recombination on the two chromosomes containing exo $70^{M B 04553}$ and C57. We first crossed the homozygous C57-GAL4 line to exo70 $0^{M B 04553} / T M 6 B, S b T b$, and selected female progenies without balancer. These female flies were then crossed with $\mathrm{Dr} / \mathrm{TM} 6 \mathrm{~B}, \mathrm{Sb} \mathrm{Tb}$ males. We selected larvae that contained $T M 6 B, S b T b$ and exo70 $0^{M B 04553}$. Larvae that contained exo $70^{M B 04553}$ were easily observed because the Mi[ET1] transposon exhibits GFP expression in the CNS and at the larval anal pad. Mi[ET1] does not contain a mini-white gene, whereas the C57-GAL4 does. Thus, when the larvae developed into adult flies, we chose male flies with eye color. The male flies were then crossed with $\mathrm{Dr} / \mathrm{TM} 6 \mathrm{~B}, \mathrm{Sb} \mathrm{Tb}$ to establish a few independent $C 57$ exo $70^{M B 04553}$ stocks. One of the lines was then verified using genomic PCR (see below) before being used in our experiments. Both the exo70 gene and the elav ${ }^{G S}$ are located on the third chromosome; we performed recombination on the chromosomes containing exo70 ${ }^{M B 04553}$ and $\operatorname{elav}^{G S}$ in the same way. We then verified the recombination by genomic PCR (see below).

Genomic PCR. Primers used to detect the KG08051 insertion were KG-chk-F1 (forward primer on genomic DNA 77 bp upstream of KG08051), 5'-GCG ATG TGT ACG ATG AGC-3' and P[SUPor-P]-F1 (forward primer on transposon, used as a reverse primer because the KG08051 is minus-oriented), and 5'-GGC ATC ATC AGC ATC AAG 
G-3'. Primers used to detect the MB04553 insertion were MB-chk-F1 (forward primer on genomic DNA 57 bp upstream of MB04553), 5' CAC TAT ATC CTC AAG TCG CTG C-3' and MiET-3'-F1 (forward primer on transposon, used as a reverse primer since $M B 04553$ is minusoriented), and 5' -CGC CTT CTA TCG CCT TCT TG-3'. Primers used to detect C57-GAL4 after recombination were Gal4-F1 5' -GCC GTC ACA GAT AGA TTG GC-3' and mini-white-5'-reverse G5'-CG AAT TAA TAG CTC CTG ATC CTC-3'. Primers used to detect elav ${ }^{G S}$-GAL4 after recombination were SV40-forward 5'-GGA AAG TCC TTG GGG TCT TC-3' and SV40-reverse, 5'-GGA ACT GAT GAA TGG GAG CA-3'. Actin primers were actin-genomic-forward $5^{\prime}$-ATG TGC AAG GCC GGT TTC G-3' and actin-genomic-reverse 5'-CAA CAC GCA GCT CGT TGT AG-3'.

$R T$-PCR. Messenger RNA was extracted from whole larvae, dissected larval CNS, or dissected larval body wall muscles (BWMs) using standard TRIzol extraction method as previously described (Li et al., 2017). Synthesis of cDNA for $+\mathrm{RT}$ reactions was performed using the ImProm-II Reverse Transcription System (Promega), whereas - RT reactions were identical except for the lack of reverse transcriptase. The $+\mathrm{RT}$ and $-\mathrm{RT}$ reactions were then diluted by twofold with water before being used for PCR. Primers used were as follows: Exo70-F, 5'-AAG GTC AAG GAT AAG GAT CGC AG-3' (on exon 6); Exo70-R 5'-GTA GAT GGG CAG TAT GTG CTC C-3' (on exon 7); Trnc-Exo70MB-F1 5' -CCA GTC GAG GAA CGC AAT AAG-3' (on exon 5); Trnc-Exo70MB-R1 5'-GCT GTT CGA ATT AAT AGT GGT TGG-3' (on MB04553 transposon); Mtrm-F 5' -CAA CGA AGG TGC ATC CCA AG-3'. Mtrm-R 5'-GAT CAG CAC GCT CCA AGG-3'. Actin-RT-PCR-F 5'-GCT GCT CTG GTC GTT GAC-3' and actin-RT-PCR-R 5'-ACA CCA TCA CCG GAG TCC-3'. Exo70-F and $\mathrm{R}$ target the $\mathrm{C}$ terminus (see Fig. $1 A$ ), and give a $162 \mathrm{bp}$ band, Trnc-Exo70MB-F1 and Trnc-Exo70MB-R1 give a 248 bp band, and Mtrm-F and R give a 357 bp band. Actin-RT-PCR-F and R give a 461 bp band.

$5 \times$ spaced $\mathrm{K}^{+}$stimulation paradigm and synaptopod analysis. All animals used in synaptopod analysis carried a copy of Tdc2-Gal4 and a copy of UAS-CD8::GFP. Animals were kept at low density. Wandering late third-instar larvae were used for experiments. Time-lapse live imaging was performed using a TCS SP8 high speed confocal microscope (in high speed mode, Leica Microsystems). Other procedure details were previously described (Ataman et al., 2008; Koon et al., 2011).

Quantification of boutons and synaptopods. The number of Type I boutons was obtained at muscles 6 and 7 of abdominal segment A3. Synaptopod number was measured from muscle 12 (A4) in dissected semiliving preparations. $n$ indicates the number of analyzed NMJs. At most, two NMJs were quantified in each animal. Other procedure details were the same as previously described (Koon et al., 2011).

Quantification of fluorescence intensity and volume. Quantification of fluorescence intensity of GluRIIA and volume of DLG and HRP were performed using IMARIS image analysis software. Quantification of fluorescence intensity in the integral protein/membrane trafficking assay was performed using ImageJ. Corrected Total NMJ Fluorescence (CTNJ) was calculated as follows: CTNJ $=$ Integrated Density - (Area of selected neuron $\times$ Mean fluorescence of background readings).

Crawling assay. Larval crawling assay was previously described (Bai et al., 2016). In brief, mid-third instar larvae were loaded onto a $2 \%$ agarose plate with a grid $\left(0.5 \mathrm{~cm}^{2}\right.$ per square $)$ placed underneath. The total number of gridlines crossed by the animals in 1 min was then counted. $n$ indicates the number of larvae analyzed. The procedure of larvae starvation was previously described (Koon et al., 2011). Briefly, it was performed by loading $10-15$ assayed larvae into a wet $10 \mathrm{~mm}$ tissue culture dish. The dish is sealed with Parafilm with a few small pin holes for air flow and then incubated at $25^{\circ} \mathrm{C}$ for $2 \mathrm{~h}$. The larvae are then subjected to crawling assay again.

Immunohistochemistry and confocal microscopy. Wandering third instar larvae were dissected in $\mathrm{Ca}^{2+}$-free EGTA hemolymph-like saline and fixed for $15 \mathrm{~min}$ in 4\% PFA as previously described (Budnik et al., 1996). For the analysis of neurite arborization in adult CNS, freshly eclosed adult flies were collected within $10 \mathrm{~min}$ of eclosion. The entire CNS was dissected in $\mathrm{Ca}^{2+}$-free EGTA hemolymph-like saline, fixed for $30 \mathrm{~min}$ in $4 \%$ PFA, and immunostained with anti-CD8 $\alpha$ at 1:100 (Thermo Fisher
Scientific, MA1-10301) overnight at $4^{\circ} \mathrm{C}$. For the immunostaining of Exo70, samples were fixed in Bouin's fixative for $15 \mathrm{~min}$, blocked with $0.3 \%$ BSA in $0.1 \mathrm{~m}$ phosphate buffer, $\mathrm{pH} 7.2$, with $0.3 \%$ Triton X-100 at room temperature for $30 \mathrm{~min}$, and then blocked with $0.3 \% \mathrm{BSA}$ and $3 \%$ goat serum in the same buffer overnight at $4^{\circ} \mathrm{C}$ before incubating in preadsorbed Exo70 antibodies at room temperature for $2 \mathrm{~h}$. AntiExo70 ${ }^{\text {Maguro }}$ and anti-Exo70 ${ }^{\text {Sake }}$ were preadsorbed at $4{ }^{\circ} \mathrm{C}$ overnight in Bouin's-fixed larval preparations of exo70 KG08051 $/ D f(3 L) B S C 815$ (15 larvae per $500 \mu \mathrm{l})$ at concentrations of 1:250 and 1:500, respectively, and used at final concentrations of 1:500 and 1:1000, respectively. Other antibodies and their concentrations used were anti-CD8 $\alpha$ 1:100 (Thermo Fisher Scientific, MA1-10301), anti-HRP-AlexaFluor-488/-594/-647 1:400/1:300/1:400 (Jackson ImmunoResearch Laboratories), anti-FasII 1:50 (DSHB catalog \#1D4, RRID:AB_528235), anti-Syt1 1:100 (DSHB catalog \#3H2 2D7, RRID:AB_528483), anti-Bruchpilot 1:100 (DSHB catalog \#nc82, RRID:AB_2314866), anti-Futsch 1:100 (DSHB catalog \#22C10, RRID:AB_528403), anti-GluRIIA 1:100 (DSHB catalog \#8B4D2 (MH2B), RRID:AB_528269), and anti-tyramine $\beta$ hydroxylase (TBH) 1:200 (Koon et al., 2011). Secondary antibodies conjugated to AlexaFluor-488/-594/-647 (Abcam) were used at a concentration of 1:200. Confocal micrographs were captured using a TCS SP8 high speed confocal microscope (Leica Microsystems).

Electrophysiology and analysis. Electrophysiology procedures had been previously described (Yeates et al., 2017).

Western blot analysis. For the preparation of protein samples, flies were raised at $25^{\circ} \mathrm{C}$ and adult heads were collected. Proteins were extracted from adult heads using SDS sample buffer (100 mM Tris-HCl, pH 6.8, 2\% SDS, $20 \%$ glycerol, $5 \% \beta$-mercaptoethanol, $0.02 \%$ bromophenol blue). All protein samples were boiled at $99^{\circ} \mathrm{C}$ for $10 \mathrm{~min}$, before immunoblot analysis. Primary antibodies used were anti-p-c-Jun 1:500 (Cell Signaling Technology, catalog \#9261L, RRID:AB_2130159), anti-c-Jun 1:1000 (Cell Signaling Technology, catalog \#2315, RRID:AB_490780), anti-p-JNK 1:1000 (Cell Signaling Technology, catalog \#9251, RRID: AB_331659), and anti-JNK 1:1000 (Cell Signaling Technology, catalog \#9252, RRID:AB_2250373). Secondary antibodies used were goat-antirabbit 1:5000 (11-035-045) and goat-anti-mouse 1:10,000 (115-035-062) from Jackson ImmunoResearch Laboratories.

Statistical analysis. For comparisons between three or more sample groups, an ANOVA with Tukey post hoc test was performed. For pairwise comparisons, a Student's $t$ test was used. All histograms indicate mean \pm SEM. All experiments were performed three times independently.

\section{Results}

Characterization of exo $70^{K G 08051}$ and $\operatorname{exo} 70^{M B 04553}$ mutant alleles The Drosophila exo70 gene is located at 66C11 on the third chromosome. Its locus encompasses the matrimony ( $\mathrm{mtrm}$ ) gene, which is located on the third intron of exo70 (Fig. 1A). To study the functions of Exo70, we acquired two fly strains from Bloomington Drosophila Stock Center containing $P$ element transposons inserted in the exo70 locus. KG08051 is a suppressor of Hairy-wing-based $P$ element $(P[S U P o r-P])$ inserted in the third intron of the exo70 gene, whereas MB04553 is a Minos-based $P$ element $(M i[E T 1])$ inserted in the sixth exon (Fig. 1A). First, we confirmed that these lines indeed possessed the specific insertions at the expected locations of the exo70 locus using genomic PCR (Fig. 1B). After that, using RT-PCR, we demonstrated that both KG08051 and MB04553 produced no detectable exo70 transcripts (using a pair of primers against a region that spans the sixth and seventh exon) (Fig. $1 A, C$ ), thereby validating both strains to be exo70 mutant alleles. Because KG08051 is in proximity to the 5'UTR of $m$ trm, we further performed RT-PCR to determine whether $m$ trm transcripts would be disrupted by this transposon. Indeed, $m$ trm transcription was disrupted by KG08051, but not by $M B 04553$ (Fig. 1C). The $m$ trm gene encodes for a protein involved in the regulation of meiotic cell cycle and homologous chromosome segregation (Harris et al., 2003). exo $70^{K G 08051}$ is a homozygous viable allele, with both male and female sterility. 
A
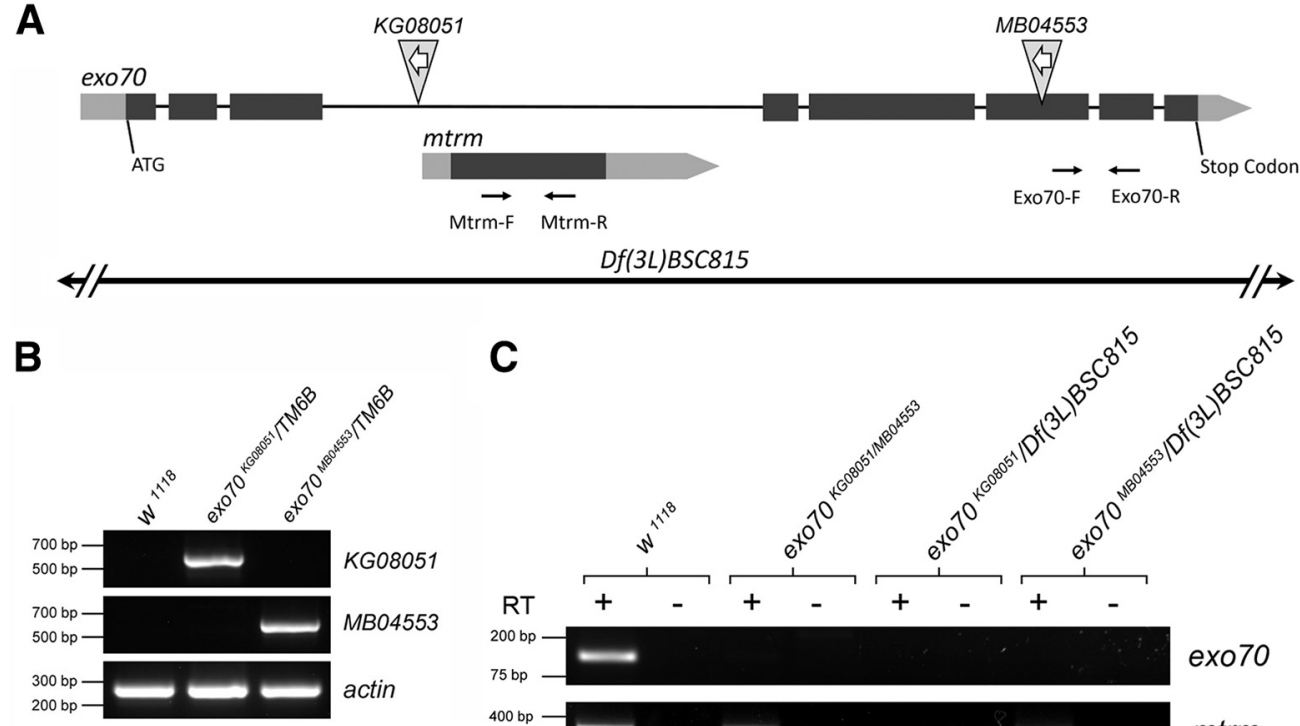

C


Figure 1. Characterization of Drosophila mutants of exo70. A, The exo 70 locus encompasses the mtrm locus. Light gray represents UTR. Dark gray represents ORF. KG08051 is a $P$ element located on the third intron of exo70. MB04553 is a P element located on exon 6. White arrows inside the triangles indicate that both $P$ elements are in the "minus" orientation. Black arrows indicate primers designed for RT-PCR. B, Genomic PCR using primers specific to the exo70 locus and the sequence of the corresponding Pelement verified the insertions of KG08051 and MB04553. C, RT-PCR analysis of using Ex070-F and R primers and Mtrm-F and R primers. $m$ trm transcription was disrupted by the exo $70^{K G 08051}$ allele, but not affected by the exo $70^{M B 04553}$ allele. $D, R T-P C R$ analysis using primers specific to the predicted truncated exo 70 transcripts in the ex0 $70^{\mathrm{MBO} 04553}$ allele. $\boldsymbol{E}$, Quantification of locomotor activity. $n$ indicates the number of analyzed larvae. $F-I$, Confocal micrographs of NMJs on muscles 6 and 7 of segment A3. Anti-HRP marks presynaptic boutons. Anti-Discs large (DLG) marks the postsynaptic density. Scale bar, $15 \mu \mathrm{m} . \boldsymbol{F}, w^{1118}$ control. G, exo70 ${ }^{K G 08057 / M B 04553}$.

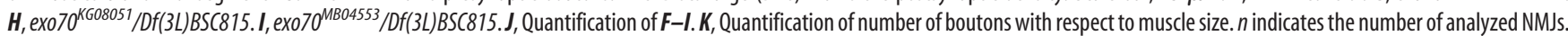
All experiments were performed three times independently. Statistical analysis was performed using ANOVA. Error bars indicate SEM. ${ }^{* *} p<0.01,{ }^{* * *} p<0.001$. 
exo70 ${ }^{M B 04553}$ is homozygous lethal, but $\operatorname{exo}^{\mathrm{MB04553}} / \mathrm{Df}(3 \mathrm{~L}) \mathrm{BSC} 815$ is viable, indicating that the lethality of the exo70 $0^{M B 04553}$ allele is likely due to secondary mutations in the fly strain. In silico prediction suggested that the MB04553 insertion in the sixth exon would introduce a stop codon 182 nucleotides downstream of the insertion site. $D f(3 L) B S C 815$ is a deficiency line carrying a large deletion in the 66C3-66D4 genomic region, which removes exo70, mtrm, and other genes in the region. To investigate whether the MB04553 insertion could potentially result in truncated Exo70 transcripts, we performed RT-PCR using primers specifically designed against only the predicted truncated transcript. Indeed, we detected the truncated transcripts in exo70 ${ }^{M B 04553}$ animals (Fig. 1D). However, to avoid disrupting both copies of $m$ trm and to avoid phenotypes caused by secondary mutations, we performed our analysis in this study mostly on the transheterozygous exo70 KG08051/MB04553. Similar to the homozygous exo70 KG08051/KG08051, transheterozygous exo70 KG08051/MB04553 mutants are also both male and female sterile, indicating that this phenotype is specifically caused by the loss of exo70. In addition, we observed that exo70 KG08051/MB04553, exo70 $1304553 /$ $D f(3 L) B S C 815$, and exo70 ${ }^{K G 08051} / D f(3 L) B S C 815$ all exhibited a slow growth phenotype. Larvae of these genotypes hatched $\sim 24 \mathrm{~h}$ later than $w^{1118}$ controls. After hatching, they took an additional $24 \mathrm{~h}$ compared with the $w^{1118}$ control to reach the wandering third instar larval stage. Considering these developmental delays, the pupation of exo 70 mutants was $\sim 48$ h later than $w^{1118}$ controls.

\section{Presynaptic and postsynaptic Exo70 is necessary for normal locomotor activity, but only presynaptic Exo70 is required for normal synaptic growth}

To investigate whether exo70 mutants have behavioral defects, we performed a crawling assay on the third instar larvae of exo70 mutants and controls. We found that the transheterozygous exo70 KG08051/MB04553 larvae displayed reduced locomotor activity compared with control animals (Fig. $1 E)\left(18.25 \pm 0.47\right.$ in $w^{1118}$ vs $13.80 \pm 0.67$ in exo70 $0^{K G 08051 / M B 04553}, p=0.0000007 ; 18.46 \pm 0.51$ in exo70 ${ }^{K G 08051} /+$ vs $13.80 \pm 0.67$ in exo70 ${ }^{K G 08051 / M B 04553}, p=$ $0.0000009 ; 17.77 \pm 0.41$ in exo70 $0^{M B 04553 /+}$ vs $13.80 \pm 0.67$ in exo70 KG08051/MB04553,$p=0.0000070)$. Defective locomotion may be caused by the impairment of higher brain functions or the neuromusculature. Thus, we examined the NMJs of exo70 mutants and found that the mutant animals had decreased synaptic bouton numbers (Fig. $1 F-J)\left(82.2 \pm 2.7\right.$ in $w^{1118}$ vs $66.2 \pm 2.9$ in exo70 KG08051/MB04553 $p=0.000250 .80 .5 \pm 3.8$ in exo $p 0^{K G 08051} /^{+}$ vs $66.2 \pm 2.9$ in exo70 $0^{K G 08051 / M B 04553}, p=0.009283 ; 79.2 \pm 2.6$ in

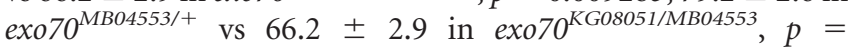
$0.002169 ; 82.2 \pm 2.7$ in $w^{1118}$ vs $62.5 \pm 2.6$ in exo70 $\stackrel{\text { KG08051/ }}{\rho}$ $D f(3 L) B S C 815, p=0.000002 ; 78.1 \pm 3.0$ in $D f(3 L) B S C 815 /+$ vs exo70 $0^{K G 08051} / D f(3 L) B S C 815, p=0.000373 ; 82.2 \pm 2.7$ in $w^{1118}$ vs $65.7 \pm 2.4$ in exo70 $0^{M B 04553} / D f(3 L) B S C 815, p=0.000028 ; 78.1 \pm$ 3.0 in $D f(3 L) B S C 815 /+$ vs exo70 $0^{M B 04553} / D f(3 L) B S C 815, p=$ $0.002664)$. Because exo70 mutants have a developmental delay, it is necessary to examine the growth of both the motor neuron terminals as well as the BWMs. Thus, we normalized the bouton numbers to the size of the BWMs and found that exo70 mutants still displayed a synaptic undergrowth $\left(1.94 \pm 0.07\right.$ in $w^{1118}$ vs $1.54 \pm 0.08$ in exo70 $0^{K G 08051 / M B 04553}, p=0.00066 ; 1.94 \pm 0.07$ in $w^{1118}$ vs $1.56 \pm 0.12$ in exo $70^{K G 08051} / D f(3 L) B S C 815, p=0.00243$; $1.94 \pm 0.07$ in $w^{1118}$ vs $1.56 \pm 0.09$ in exo $70^{M B 04553} / D f(3 L)$ $B S C 815, p=0.00027)$.

To determine whether these phenotypes resulted from the loss of Exo70 functions in the motor neurons or muscles, we sought to knock down exo70 separately in these cell types. First, using
RT-PCR, we confirmed that exo70 is expressed in both the CNS and BWMs (Fig. 2A). Next, we tested the efficiency of two independent exo70 RNAi fly lines (UAS-exo70 GD12140 $-d s R N A$ and $U A S$-exo70 ${ }^{K K 101154}-d s R N A$ ) by expressing exo70 dsRNAs in the CNS using the pan-neuronal driver elav-GAL4. We dissected the CNS of the wandering third instar larvae and performed RTPCR. Our results showed that exo70 was knocked down by these dsRNA constructs (Fig. 2B). We then crossed the dsRNA fly lines to either C380-GAL4 (motor neuron driver) or C57-GAL4 (muscle driver) and performed crawling assay and NMJ morphology analysis. We found that the knockdown of exo70 expression in either motor neurons or muscles was sufficient to induce decreased crawling speed (Fig. 2C) (18.75 \pm 0.50 in C380 control vs $14.73 \pm 0.38$ in $C 380>\operatorname{exo} 00^{G D 12140}-d s R N A, p=0.00000004$; $18.75 \pm 0.50$ in $C 380$ control vs $14.73 \pm 0.38$ in C380> exo70 $0^{K K 101154}$-dsRNA, $p=0.00000001 ; 18.68 \pm 0.36$ in C57 control vs $13.31 \pm 0.41$ in $C 57>\operatorname{exo} 70^{G D 12140}-d s R N A, p=$ $0.00000001 ; 18.68 \pm 0.36$ in C57 control vs $15.00 \pm 0.49$ in $\left.C 57>\operatorname{exo} 70^{K K 101154}-d s R N A, p=0.00000017\right)$. However, only the knockdown of exo70 expression in motor neurons resulted in the reduction of bouton numbers (Fig. 2D-G) (18.75 \pm 0.50 in C380 control vs $14.73 \pm 0.38$ in $C 380>$ exo $70^{G D 12140}-d s R N A, p=$ $0.00010979 ; 18.75 \pm 0.50$ in $C 380$ control vs $14.73 \pm 0.38$ in C380>exo70 $\left.{ }^{K K 101154}-d s R N A, p=0.00000008\right)$.

To rescue the synaptic growth phenotype of exo70 mutants, we generated UAS-exo70 transgenic line. We first confirmed the overexpression of exo70 in the UAS-exo70 transgenic line using RT-PCR (Fig. 2H). Afterward, we attempted to rescue the bouton reduction phenotype in exo70 KG08051/MB04553 mutants by overexpressing exo70 in either motor neurons or muscles. We found that the overexpression of exo70 in motor neurons rescued the bouton numbers in exo70 KG08051/MB04553 mutants, whereas the overexpression in the BWMs did not (Fig. $2 I)(62.6 \pm 2.7$ in C380 driver, exo70 $0^{K G 08051 / M B 04553}$ vs $74.5 \pm 2.5$ in C380>UAS exo70, exo70 $\left.{ }^{K G 08051 / M B 04553}, p=0.004052\right)$. Thus, during development, Exo70 is required cell autonomously in motor neurons for normal synaptic growth.

\section{Presynaptic Exo70 is present in synaptic boutons at the NMJ}

To determine the subcellular localization of Exo70, we independently generated two antibodies (anti-Exo70 Maguro and antiExo70 ${ }^{\text {Sake }}$ ) against the first 400 amino acids of Drosophila Exo70, and performed immunohistochemistry at the NMJ. In $w^{1118}$ control animals, anti-Exo70 ${ }^{\text {Maguro }}$ was detected in the synaptic boutons and BWMs (Fig. 3A). In exo70 $0^{K G 08051} / \mathrm{Df}(3 \mathrm{~L}) \mathrm{BSC} 815$ animals, the signals in muscle remained, but the signal in boutons was not detected (Fig. 3B). This could be observed more clearly in magnified confocal micrographs in Figure $3 C, D$; the absence of signals in the boutons of exo70 KG08051 $/ D f(3 L) B S C 815$ animals indicated that the signals in the boutons of control animals were specific to Exo70 (Fig. 3C,D). The Exo70 staining persisted in the boutons of exo70 KG08051/MB04553 mutants (Fig. 3E), suggesting exo $70^{M B 04553}$ to be a hypomorphic allele with truncated proteins. Overexpression of exo70 in motor neurons resulted in increased immunostaining (Fig. $3 F$ ). In exo70 knockdown animals using both $C 380$ (motor neuron driver) and C57 (muscle driver), the anti-Exo70 Maguro immunostaining in boutons was significantly reduced (Fig. 3G-I). In all the genotypes tested, we noticed that the immunostaining in the muscles persisted, including exo70 mutants and knockdown animals. Thus, even though Exo70 is expressed in BWMs (Fig. $2 \mathrm{~A}$ ), the anti-Exo70 ${ }^{\text {Maguro }}$ signals in the muscles might not be Exo70-specific. Because anti-Exo70 Maguro 
A
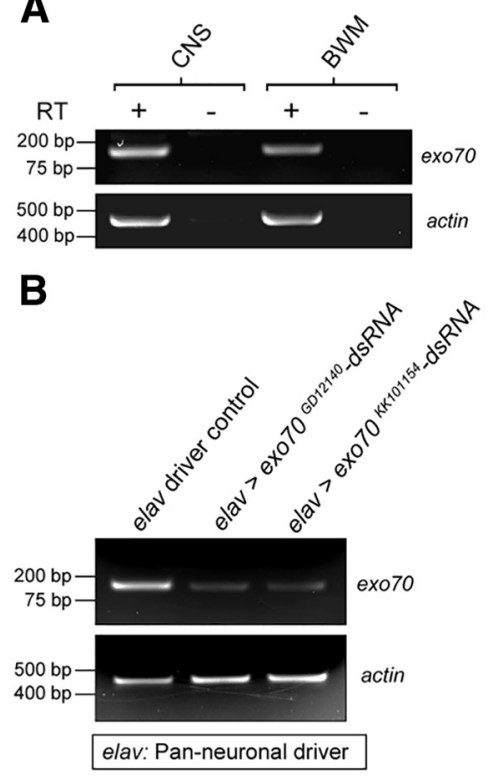

C



G

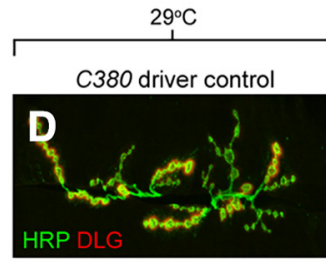

$C 380>\operatorname{ex070} 0^{6012140}-d s R N A$

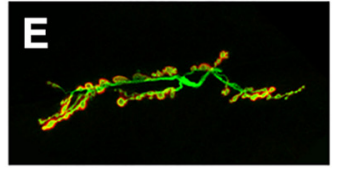

C380 > exo70 ${ }^{K K 101154-d s R N A}$

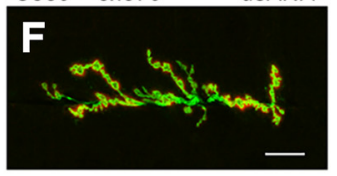



H

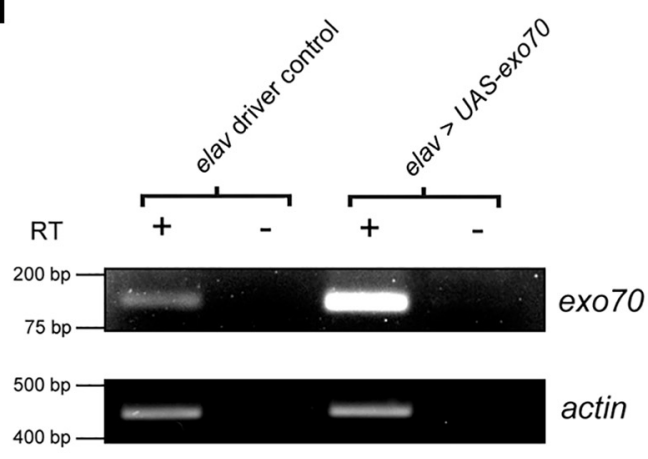



Figure 2. Presynaptic and postsynaptic Ex070 is required for normal locomotor activity, whereas only presynaptic Exo70 is required for normal synaptic growth. $\boldsymbol{A}$, RT-PCR analysis of exo70 expression in the CNS and BWMs. exo70 was expressed in both the CNS and BWM of $w^{1118}$ larvae. B, RT-PCR of exo70 from the CNS of driver control and pan-neuronal exo70 knockdown larvae, demonstrating exo70 to be knocked down by both dsRNA constructs. C, Quantification of locomotor activity. $n$ indicates the number of analyzed larvae. D-F, Confocal micrographs of NMJs. D, C380 driver control, $\boldsymbol{E},\left(380>\right.$ exo $70^{6 D 12140}$-dsRNA.F, C380 $>$ exo $70^{K K 101154}$-dsRNA. Scale bar, $15 \mu \mathrm{m}$. G, Quantification of $\boldsymbol{D}-\boldsymbol{F}$. H, RT-PCR using the CNS of control and animals overexpressing UAS-exo70 pan-neuronally. I, GFP expression in the CNS and the larval anal plate in a late third instar larva in the presence of exo70 ${ }^{\text {MB04553 }}$. Scale bar, $200 \mu \mathrm{m} .{ }^{*} p<0.05,{ }^{* *} p<0.01$, ${ }^{* * *} p<0.001$.

and anti-Exo70 ${ }^{\text {Sake }}$ gave identical staining patterns (Fig. $3 J-L$ ), we used anti-Exo $70^{\text {Maguro }}$ for the rest of our analysis in this study.

exo70 mutants have a lower density of GluRIIA-containing subset of glutamate receptors and decreased mEPSP amplitude

To identify synaptic molecules that could be regulated by Exo70, we used antibodies against variety of synaptic proteins to look for changes in expression level or localization in exo70 mutants. We examined Bruchpilot (Wagh et al., 2006), Dlg (Parnas et al., 2001), Futsch (Fujita et al., 1982), and Synaptotagmin (Dubuque et al., 2001), and did not observe any apparent change (data not shown). In contrast, we noticed that exo70 mutants had more discrete GluRIIA clusters in boutons (Fig. $4 A, B$ ). We quantified the total GluRIIA fluorescence intensity with respect to Dlg volume in Type Ib boutons and found that exo70 mutants had significantly lower GluRIIA density (Fig. $4 C)\left(0.82 \pm 0.10\right.$ in $w^{1118}$ control vs $0.51 \pm 0.05$ in exo70 1 KG08051/MB04553,$p=0.00589$. $0.82 \pm 0.10$ in $w^{1118}$ control vs $0.44 \pm 0.06$ in exo $70^{K G 08051} /$ $D f(3 L) B S C 815, p=0.00113)$. To ensure that the volume of the postsynaptic density in exo70 mutants did not differ from that of control animals, we quantified the Dlg volume with respect to 

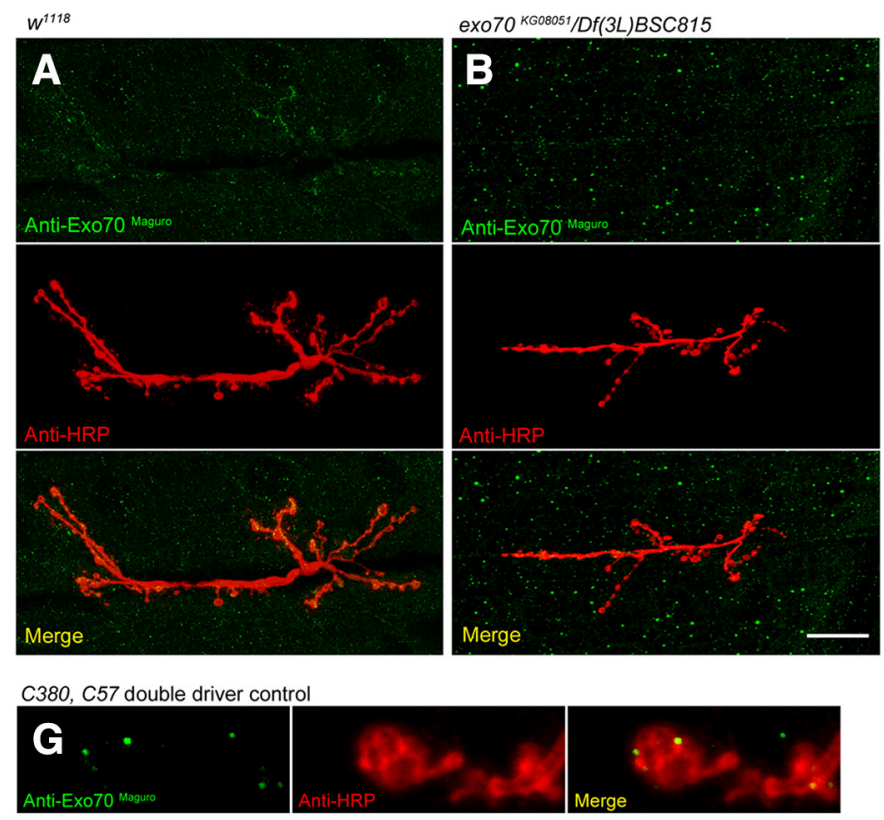

C380, C57 > exo70 $0^{6012140}$-dsRNA
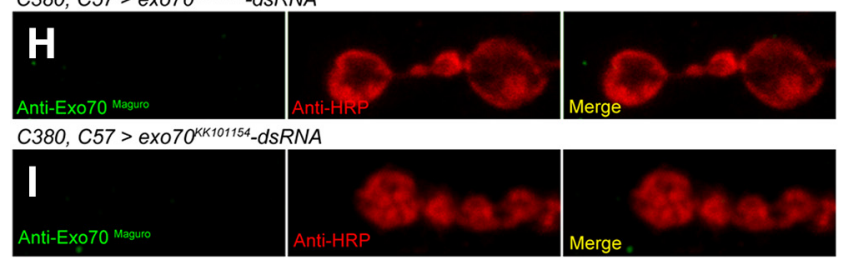

$w^{1118}$

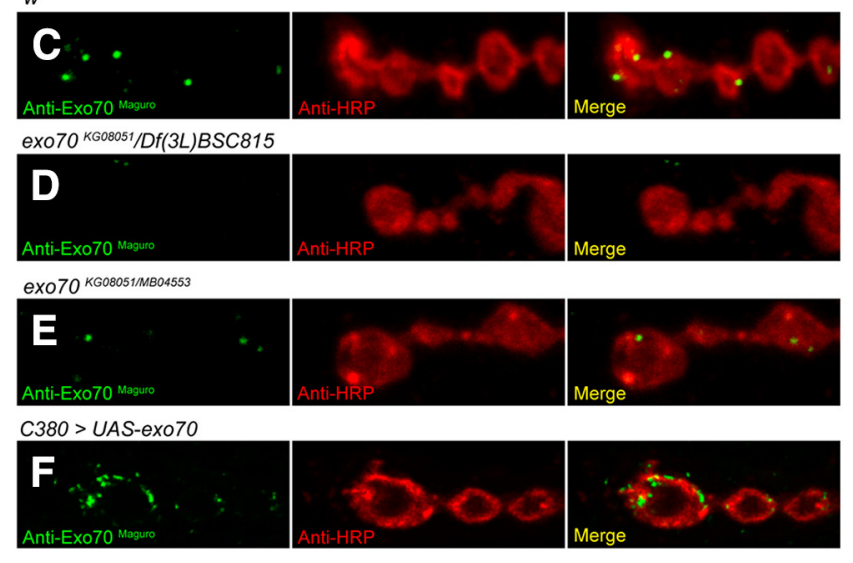

C380, C57 double driver control



C380, C57 > exo70 $0^{6012140}$-dsRNA

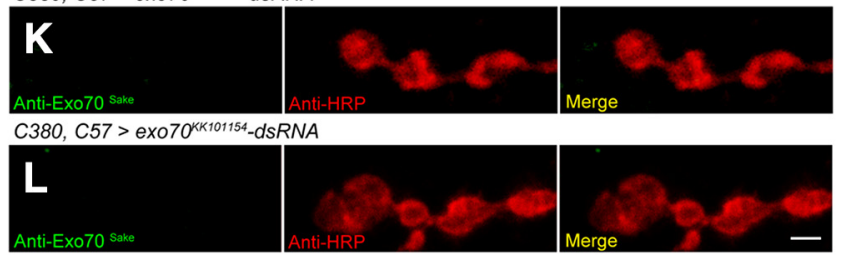

Figure 3. Exo70 is localized at synaptic boutons at the NMJ. Confocal micrographs of larval NMJs on muscles 6 and 7 of segment A3, colabeled with anti-HRP and anti-Exo70 Maguro $(\boldsymbol{A}-\boldsymbol{I})$ or anti-HRP and anti-Exo70 Sake $(\boldsymbol{J}-\boldsymbol{L})$. Low-magnification confocal micrographs showing the entire NMJ of $(\boldsymbol{A}) w^{1118}$ and $(\boldsymbol{B})$ exo70 ${ }^{K G 08051} / \mathrm{Df}(3 \mathrm{~L}) B S C 815$. Scale bar, $2.5 \mu \mathrm{m}$. High-magnification confocal micrographs of $(\boldsymbol{C}) w^{1118},(\boldsymbol{D})$ exo70 ${ }^{K G 08051} / \mathrm{Df}(3 \mathrm{~L}) B S C 815$, (E) exo70 ${ }^{K G 08051 / M B 04553},(\boldsymbol{F})$ C380 > UAS-exo70, (G, J) C380,C57 double driver Control, (H, K) C380,C57> exo70 GD12140 -dsRNA, and $(\boldsymbol{I}, \boldsymbol{L})\left(380, C 57>\right.$ exo $0^{K K 101154}$-dsRNA. Scale bar, $2.5 \mu \mathrm{m}$. All experiments were performed three times independently.

HRP volume in exo70 mutants and found no significant difference (Fig. $4 D)\left(2.26 \pm 0.07\right.$ in $w^{1118}$ control vs $2.09 \pm 0.15$ in exo70 $0^{K G 08051 / M B 04553}, p=0.4855 .2 .26 \pm 0.07$ in $w^{1118}$ control vs $2.05 \pm 0.10$ in exo70 $\left.{ }^{K G 08051} / D f(3 L) B S C 815, p=0.3580\right)$. These results demonstrated that exo70 mutants have a lower density of GluRIIA-containing subset of glutamate receptors.

Interestingly, in $w^{1118}$ control animals, Exo70 and Bruchpilot were found juxtaposed to each other, indicating that the exocyst complex could be involved in activity-related cellular processes near the active zones (Fig. $4 E, F$ ). This is an intriguing observation, as it had been shown that exocyst complex subunits, such as Sec5, are not involved in the release of synaptic vesicles in neurons in Drosophila (Murthy et al., 2003). To test whether exo70 mutations would affect synaptic neurotransmission, we performed electrophysiological analyses on exo70 mutants by measuring the amplitude and frequency of mEPSPs and amplitudes of EPSPs. We found that both exo70 $0^{K G 08051 / M B 04553}$ and exo70 $0^{K G 08051} / D f(3 L) B S C 815$ had significantly lower mEPSP amplitude than the $w^{1118}$ control (Fig. 4G,I) $(1.14 \pm 0.06 \mathrm{mV}$ in $w^{1118}$ control vs $0.77 \pm 0.04 \mathrm{mV}$ in exo70 $0^{K G 08051 / M B 04553}, p<$ $0.0001 ; 1.14 \pm 0.06 \mathrm{mV}$ in $w^{1118}$ control vs $0.59 \pm 0.04 \mathrm{mV}$ in exo70 $\left.{ }^{K G 08051} / \mathrm{Df}(3 L) B S C 815, p<0.0001\right)$. Furthermore, exo70 ${ }^{K G 08051} /$ $D f(3 L) B S C 815$ exhibited significantly lower mEPSP frequency than control (Fig. $4 J)\left(2.82 \mathrm{~Hz} \pm 0.23\right.$ in $w^{1118}$ control vs 1.71 $\mathrm{Hz} \pm 0.24$ in exo70 $\left.0^{K G 08051} / D f(3 L) B S C 815, p=0.0169\right)$. exo $70^{K G 08051 / M B 04553}$, which contains the hypomorphic allele exo $70^{M B 04553}$, also showed a reduced frequency of mEPSPs than that of the control, but the difference was not statistically significant (Fig. $4 J)\left(2.82 \mathrm{~Hz} \pm 0.23\right.$ in $w^{1118}$ control vs $2.26 \mathrm{~Hz} \pm 0.36$ in exo70 $\left.{ }^{K G 08051 / M B 04553}, p=0.4239\right)$. No significant difference in EPSP amplitude was found between the $w^{1118}$ control and exo70 ${ }^{K G 08051 / M B 04553}$ (Fig. $\left.4 H, K\right)\left(28.28 \pm 1.62 \mathrm{mV}\right.$ in $w^{1118}$ control vs $26.93 \pm 1.26 \mathrm{mV}$ in exo70 $\left.{ }^{K G 08051 / M B 04553}, p=0.8271\right)$, although exo70 ${ }^{K G 08051} / D f(3 L) B S C 815$ did demonstrate a small but significant decrease of EPSP amplitude compared with control (Fig. $4 H, J)\left(28.28 \pm 1.62 \mathrm{mV}\right.$ in $w^{1118}$ control vs $22.17 \pm 2.01$ $\mathrm{mV}$ in exo70 $\left.{ }^{K G 08051} / \mathrm{Df}(3 \mathrm{~L}) \mathrm{BSC} 815, p=0.0384\right)$.

The decrease in mEPSP amplitude in exo70 mutants was consistent with the loss of GluRIIA-containing receptors (Petersen et al., 1997; Yeates et al., 2017). Additionally, when GluRIIAcontaining receptors are lost at the NMJ, there is an expected homeostatic increase in quantal content (QC) (Yeates et al., 2017). We observed an obvious homeostatic increase of QC in exo70 mutants. For QC, exo70 mutants were significantly higher than that of the control (Fig. $4 L)\left(25.46 \pm 1.45\right.$ in $w^{1118}$ control vs $35.87 \pm 2.12$ in exo $70^{K G 08051 / M B 04553}, p=0.0443 .25 .46 \pm 1.45$ in $w^{1118}$ control vs $39.67 \pm 4.02$ in exo $70^{K G 08051} / D f(3 L) B S C 815, p=$ $0.0023)$. Overall, we showed a profound decrease in quantal size in all exo70 mutants, which is consistent with our observed decrease in glutamate receptor density (Fig. 4A-C). mEPSP frequency and EPSP amplitudes were slightly decreased in exo $70^{K G 08051} / D f(3 L) B S C 815$, but otherwise relatively normal in exo $70^{K G 08051 / M B 04553}$. 

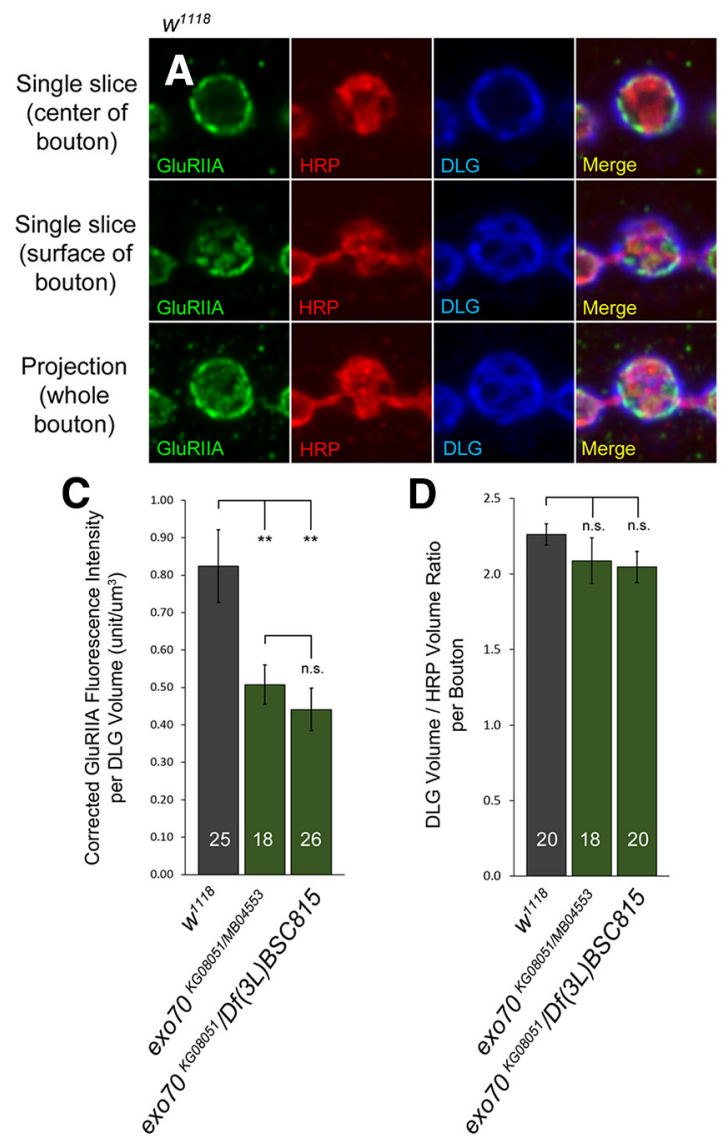

exO70 KG08051/Df(3L)BSC815
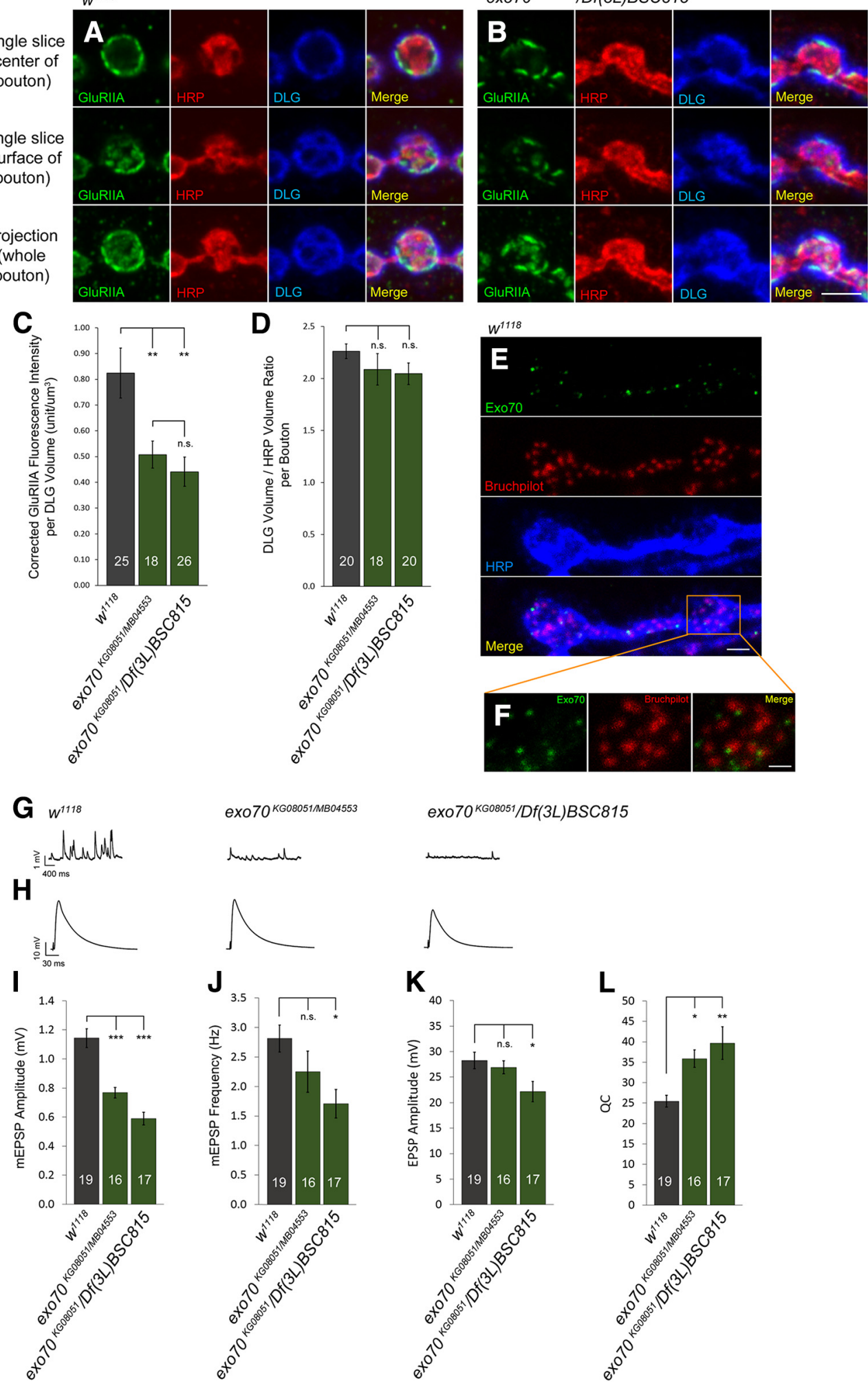

exo70 KG08051/Df(3L)BSC815
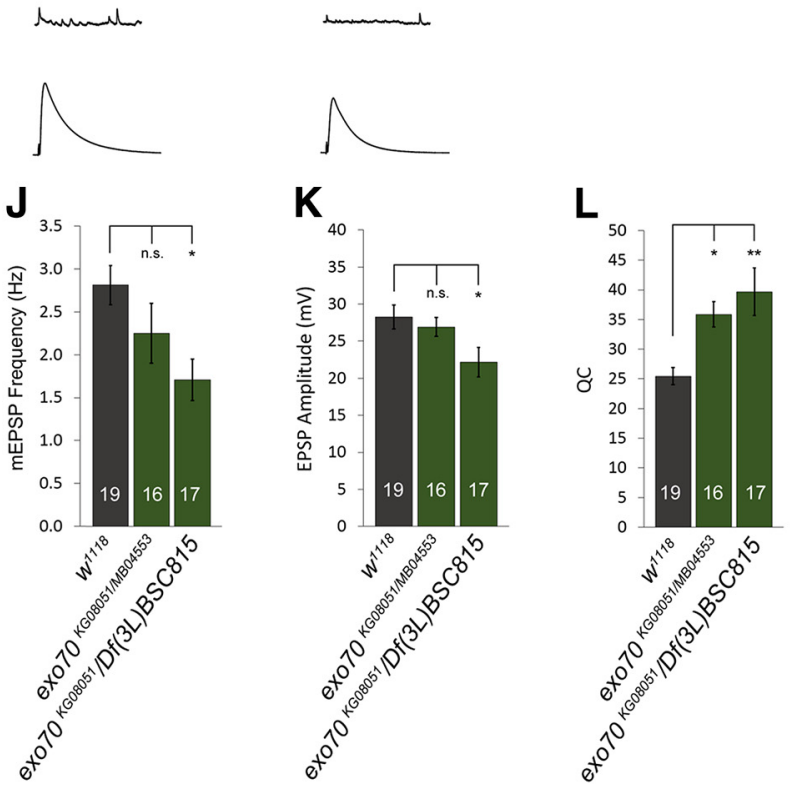

Figure 4. ex070 mutants have a lower density of GluRIIA-containing subset of glutamate receptors and decreased mEPSP amplitude. $A, B$, High-magnification confocal micrographs of a single bouton on muscles 6 and 7 of segment A3, triple-labeled with anti-GluRIIA, anti-HRP, and anti-DLG. A, $w^{1118}$. B, exo $70^{K G 08051} / D f(3 L) B S C 815$. C, Quantification of GluRIIA fluorescence intensity with respect to the volume of anti-DLG staining. D, Quantification of the volume of anti-DLG staining with respect to the volume of anti-HRP staining. $E$, $w^{1118} \mathrm{NMJ}$ triple-labeled with anti-Exo70 Maguro, anti-Bruchpilot (Nc82), and anti-HRP. Scale bar, $2.5 \mu \mathrm{m}$. $\boldsymbol{F}$, High magnification of $\boldsymbol{E}$ showing Ex070 being juxtaposed to active zones labeled by anti-Bruchpilot. Scale bar, $1.25 \mu \mathrm{m}$. $\boldsymbol{G}, \boldsymbol{H}$, Representative electrophysiological traces of (G) mEPSPs and (H) EPSPs. Calibration (mEPSPs): $400 \mathrm{~ms}, 1 \mathrm{mV}$. Calibration (EPSPs): $30 \mathrm{~ms}, 10 \mathrm{mv}$. I, Quantification of mEPSP amplitude. $\boldsymbol{J}$, Quantification of mEPSP frequency. $\boldsymbol{K}$, Quantification of EPSP amplitude. $\boldsymbol{L}$, Quantification of QC. $n$ indicates the number of analyzed boutons $(\boldsymbol{C}, \boldsymbol{D})$ and NMJs $(\boldsymbol{I}-\boldsymbol{L})$. Statistical analysis was performed using ANOVA. Error bars indicate SEM. ${ }^{*} p<0.05,{ }^{* *} p<0.01,{ }^{* *} p<0.001$. 


\section{exo70 genetically interacts with $\mathrm{ralA}$ to regulate NMJ expansion}

In mammals, the exocyst interacts with the small GTPase RalA to control exocytosis, actin cytoskeletal remodeling, and vesicle transport (Sugihara et al., 2002; Wang et al., 2004). In Drosophila, the exocyst is recruited by RalA to regulate activity-dependent growth of the postsynaptic membranes (Teodoro et al., 2013). Therefore, we sought to determine whether exo70 and ralA function in the same genetic pathway in controlling NMJ expansion. We first confirmed the expression of ralA in the CNS and BWMs using RT-PCR (Fig. 5A). Afterward, we looked for a genetic interaction in exo70, ralA double heterozygotes. We used two characterized ralA mutants in this analysis: $\mathrm{ralA}^{P G 89}$, a $P$ element inserted in the first intron of ralA that results in severe loss of function (Balakireva et al., 2006); and $\mathrm{ralA}^{E E 1}$, a mis-sense mutation that also results in severe loss of function (Eun et al., 2007; Cho and Fischer, 2011). Both exo70 $0^{K G 08051} /+\mathrm{ralA}^{P G 89} /+$ and exo $70^{K G 08051} /+\mathrm{ralA}^{E E 1} /+$ double heterozygotes showed a significant reduction in bouton numbers compared with the controls, suggesting that the two genes function together (Fig. $5 B-F$ ) $\left(77.5 \pm 2.8\right.$ in exo70 ${ }^{K G 08051 /+}$ vs $59.8 \pm 3.5$ in ralA ${ }^{\text {PG89/+ }}$; exo70 $0^{K G 08051 /+}, p=0.000493 ; 75.3 \pm 2.4$ in ralA ${ }^{P G 89 /+}$ vs $59.8 \pm$ 3.5 in ralA ${ }^{P G 89 /+}$; exo70 ${ }^{K G 08051 /+}, p=0.001030 ; 77.5 \pm 2.8$ in exo70 $0^{K G 08051 /+}$ vs $57.1 \pm 2.0$ in ralA ${ }^{E E 1 /+}$; exo $70^{K G 08051 /+}, p=$ $0.000005 ; 75.3 \pm 2.4$ in ralA ${ }^{E E 1 /+}$ vs $59.8 \pm 3.5$ in $\mathrm{ralA}^{E E 1 /+}$; exo $\left.70^{K G 08051 /+}, p=0.000037\right)$.

\section{Exo70 regulates synaptic growth in a JNK-independent manner}

RalA regulates the JNK signaling pathway (van den Berg et al., 2013). In Drosophila, the RalA-exocyst complex modulates JNKinduced apoptosis (Balakireva et al., 2006), and the JNK signaling pathway is known to be involved in NMJ growth (Sanyal et al., 2002; Etter et al., 2005; Shen and Ganetzky, 2009; Milton et al., 2011). Therefore, we wondered whether Exo70 regulates NMJ morphology via the JNK pathway. To test this hypothesis, we manipulated JNK signaling in exo70 mutants. $b s k^{D N}$ is a dominant negative form of basket (bsk), the JNK in Drosophila, whereas hep ${ }^{C A}$ is a constitutively active form of hemipterous (hep), the JNKK in Drosophila. Overexpression of $b s k^{D N}$ decreases JNK signaling, whereas hep ${ }^{C A}$ increases JNK signaling. If Exo70 regulates synaptic growth via the JNK pathway, then the presence of exo70 mutations should provide no additional effect to UAS$b s k^{D N}$ or UAS-hep ${ }^{C A}$ overexpression as the manipulation of the downstream pathway should mask over the effect of upstream components.

Indeed, overexpression of UAS-bsk $k^{D N}$ caused a decrease in synaptic boutons $(80.4 \pm 4.7$ in $C 380$ control vs $58.5 \pm 2.2$ in $\left.C 380>U A S-b s k^{D N}, p=0.000262\right)$, and the presence of exo70 mutations had no further effect on bouton numbers (Fig. $5 G$ ). However, although overexpression of UAS-hep ${ }^{C A}$ resulted in a dramatic increase of boutons in a wild-type background (68.2 \pm 2.0 in C380 control vs $117.8 \pm 5.5$ in C380>UAS-hep ${ }^{C A}, p<$ $0.000001)$, this increase was not retained in the exo70 mutant background. Indeed, the exo70 background completely blocked the JNK-induced synaptic growth (Fig. $5 H-L)(68.2 \pm 2.0$ in C380 control vs $59.5 \pm 3.0$ in C380>UAS-hep ${ }^{C A}$, exo70 ${ }^{K G 08051 / M B 04553}, p=$ $0.022596 ; 117.8 \pm 5.5$ in C380>UAS-hep ${ }^{C A}$ vs $59.5 \pm 3.0$ in C380 $>$ UAS-hep $\left.{ }^{C A}, \operatorname{exo}^{\text {KG08051/MB04553 }}, p<0.000001\right)$. These results suggested that Exo70 does not regulate synaptic growth via the JNK pathway. By contrast, the JNK pathway requires Exo70 to mediate synaptic growth.
To further confirm that Exo70 does not regulate synaptic growth via the JNK pathway, we performed Western blot analysis to examine the endogenous levels of JNK signaling. If Exo70 regulates synaptic growth via the JNK pathway, we should observe a change in the level of JNK signaling in exo70 mutants. However, no significant change was observed in the expression levels of phospho-JNK, JNK, phospho-c-Jun, and c-Jun in exo70 mutants (Fig. 5M). Our results demonstrate that Exo70 and RalA regulate synaptic growth together, but in a JNK-signaling independent manner. On the other hand, we found that JNK signalingmediated synaptic growth requires Exo70.

When UAS-hep ${ }^{C A}$ was overexpressed in motor neurons; although it resulted in a significant increase of the number of boutons, it resulted in the formation of many small boutons that are $\sim 1-2 \mu \mathrm{m}$ (Fig. $5 J, J^{\prime}$ ). Typically, Type Is boutons are between 3 and $4 \mu \mathrm{m}$ (Hoang and Chiba, 2001). Hence, these small boutons resemble more of the octopaminergic Type II boutons that regularly innervate muscles 12 and 13 , which are $\sim 1-2 \mu \mathrm{m}$ (Fig. $5 N$ ). Type II boutons do not normally innervate muscles 6 and 7 (Fig. 50 ), but it can occur at $~ 3 \%-5 \%$ in wild-type animals (Monastirioti et al., 1995). However, the postsynaptic density of Type II boutons does not express Dlg, whereas both Type Ib and Type Is boutons on muscles 6 and 7 do. We observed that the postsynaptic density of the small boutons in UAS-hep ${ }^{C A}$-overexpressing larvae did contain Dlg (Fig. 5J'). Furthermore, Type II boutons contain $\mathrm{TBH}$ (Fig. $5 N$ ), whereas these small boutons did not (Fig. $5 P)$. Thus, although these boutons were smaller than typical Type Is boutons, they were likely to be Type Is.

\section{exo70 mutants showed impaired trafficking of integral membrane proteins to the cell surface}

Apart from JNK signaling, the interaction between the exocyst complex and RalA is also known to mediate GTP-dependent exocytosis and filopodia formation (Sugihara et al., 2002; Wang et al., 2004). Thus, to elucidate the underlying mechanism of NMJ outgrowth defects in exo70 mutants, we used a characterized integral protein/membrane trafficking assay to examine the cell surface expression of the membrane-tethered CD8::GFP reporter (Murthy et al., 2003). We expressed UAS-CD8::GFP under the control of the inducible pan-neuronal driver elav ${ }^{\text {GeneSwitch }}$-GAL4 $\left(e l a v^{G S}\right)$ (Osterwalder et al., 2001) in either wild-type or exo70 KG08051/MB04553 mutant background. Given that exo70 mutants exhibit developmental delay, we matched the control animals and exo70 mutants by size. Twenty hours before the larvae reached wandering third instar stage, they were fed with food containing $200 \mu \mathrm{M}$ of the mifepristone RU486 to induce gene expression. At the wandering third instar stage, the animals were dissected and immunostained with anti-CD8 antibody in the absence of Triton X-100. We found that CD8::GFP could travel normally to synaptic boutons in the exo70 mutants. However, their transport to the cell surface was impaired (Fig. $6 A-I$ ) (for CTNF of CD8, 1,336,330 \pm 289,560 in elav $^{G S}>U A S-C D 8:: G F P$ vs $230,973 \pm 56,294$ in elav ${ }^{G S}>U A S$ $C D 8:: G F P$, exo70 KG08051/MB04553,$p=0.00592)$, thereby suggesting that the membrane fusion between secretory vesicles and the plasma membrane was disrupted.

\section{Exo70 is required for activity-induced rapid arbor extension}

Because Exo70 is required for JNK signaling-induced synaptic growth (Fig. $5 H-L$ ), we wondered whether it is also required for activity-induced synaptic growth. To test this hypothesis, we used the octopaminergic Type II NMJs to monitor the induction of synaptopods by activity. Synaptopods are dynamic filopodia-like 
A

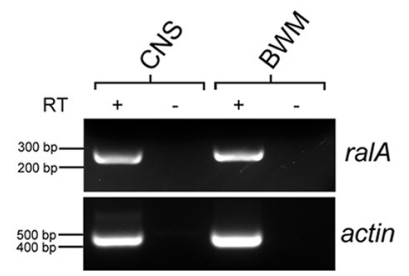

G



C380: Motor neuron driver

M

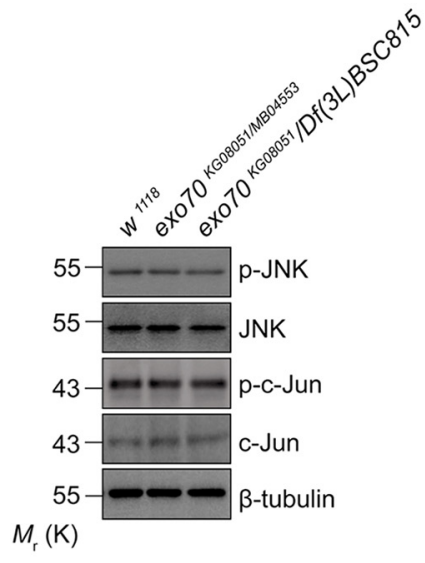

$W^{1118}$

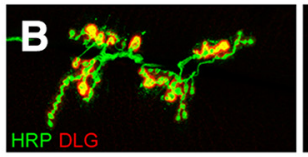

ralA ${ }^{P G 89 /+}$

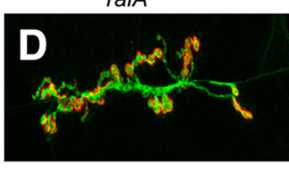

exo70 K608051/+

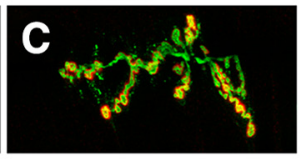

ralA ${ }^{P G 89 /+} ; e^{2} 070^{K G 08051 /+}$

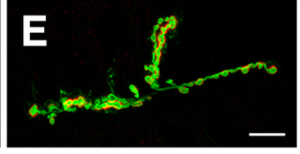

F

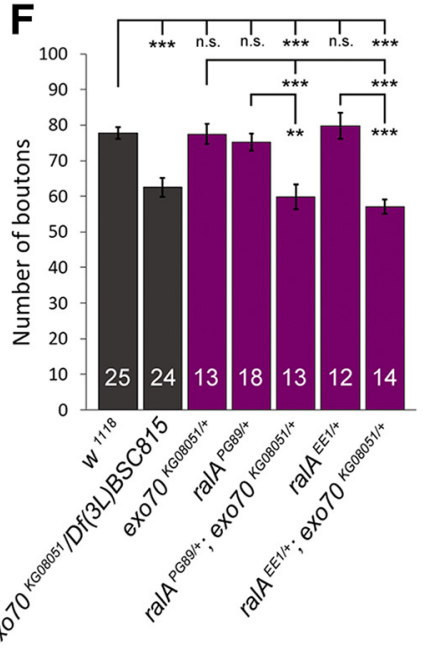

$\mathbf{L}$


C380 driver control
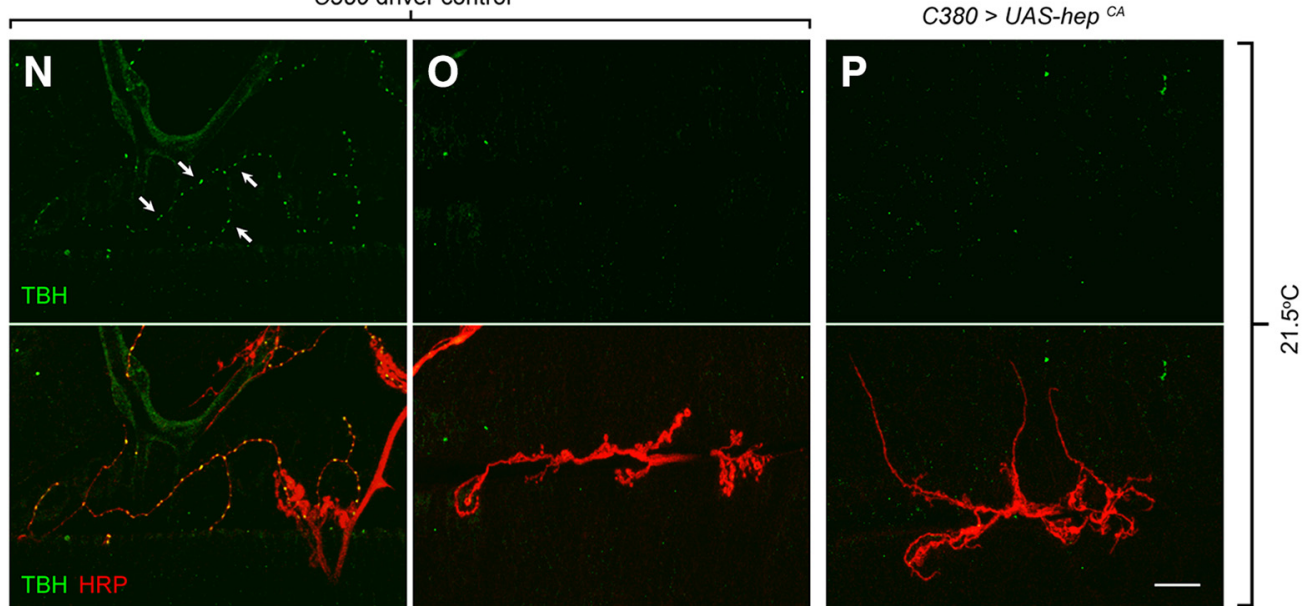

Figure 5. Exo70 genetically interacts with RalA and is necessary for JNK signaling-induced synaptic growth. $\boldsymbol{A}, \mathrm{RT}$-PCR of ralA in CNS and BWM of control larvae. $\boldsymbol{B}-\boldsymbol{E}$, Confocal micrographs of NMJs on muscles 6 and 7 of segment A3, colabeled with anti-HRP (green) and anti-DLG (red). $\boldsymbol{B}, w^{1118}$ control. $\boldsymbol{C}$, exo70 ${ }^{K G 08051}$ heterozygous mutant. $\boldsymbol{D}$, ralA ${ }^{P G 89}$ heterozygous mutant. $\boldsymbol{E}$, $\mathrm{ralA}^{P G 89}$;exo70 ${ }^{K G 08051}$ transheterozygote. Scale bar, $15 \mu \mathrm{m}$. $\boldsymbol{F}$, Quantification of $\boldsymbol{B}-\boldsymbol{E}$. G, Quantification of bouton numbers of animals overexpressing UAS-bsk ${ }^{D N}$ in wild-type or exo 70 background. $\boldsymbol{H}-\boldsymbol{K}$, Confocal micrographs of NMJs of animals overexpressing UAS-hep ${ }^{C A}$ in wild-type or exo70 background. $\boldsymbol{H}, \mathrm{C} 380$ motor neuron driver control. I, C380 driver control in exo $70^{K G 08051 / M B 04553}$ background. J, (380> UAS-hep ${ }^{C A}$. K, (380> UAS-hep ${ }^{C A}$ in exo70 ${ }^{K G 08051 / M B 04553}$ background. Scale bar, $15 \mu \mathrm{m}$. $\boldsymbol{J}^{\prime}$, High magnification of $J$. Scale bar, $15 \mu \mathrm{m}$. (Figure legend continues.) 
NO TRITON

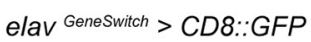
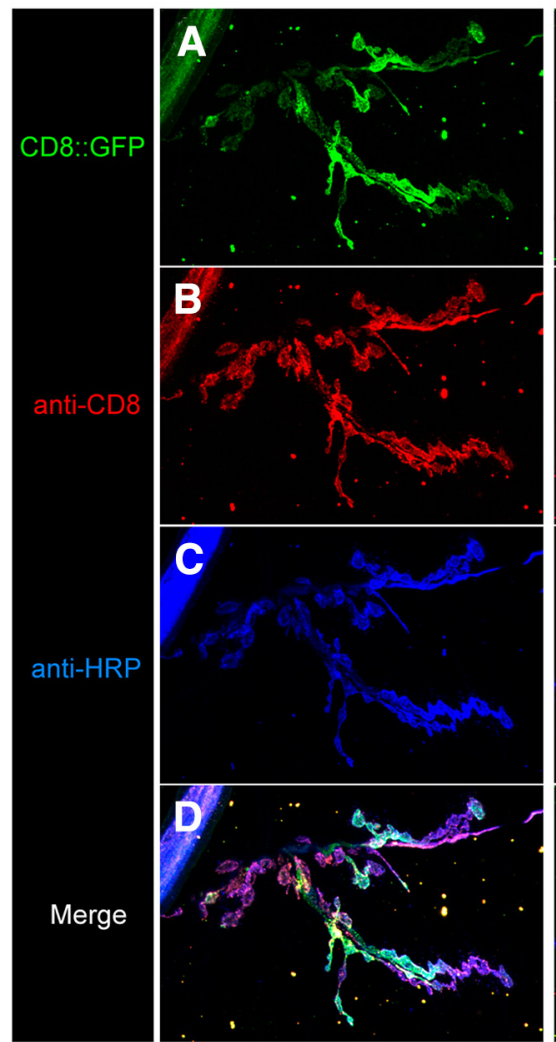

elav Geneswitch > CD8::GFP exO70 KG08051/MB04553
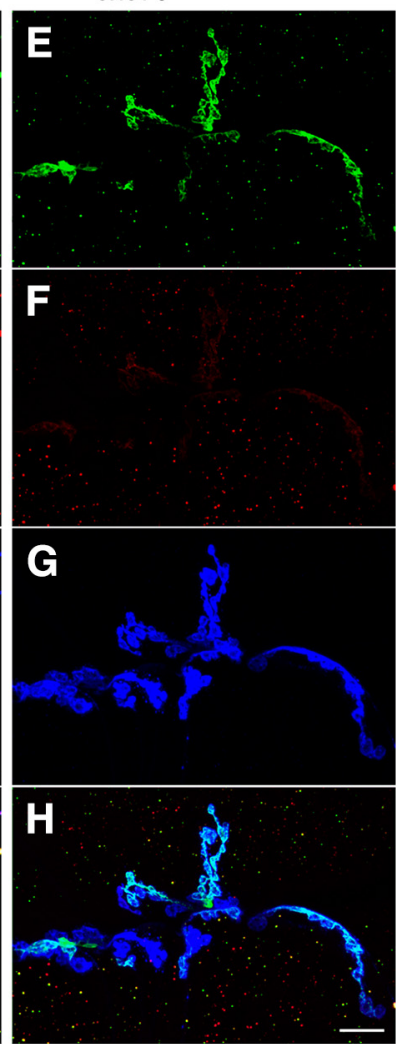


elav ${ }^{G S}:$ GeneSwitch-controlled pan-neuronal drive

Figure 6. exo70 mutants displayed impaired trafficking of integral membrane proteins to the cell surface. $A$, Genomic PCR confirmation of the presence of exo $70^{M B 04553}$ and elav ${ }^{G S}$-GAL4 in our recombinant fly line. $\boldsymbol{B}-\boldsymbol{I}$, Confocal micrographs of NMJs on muscles 6 and 7 of segment A3. elav ${ }^{G S}>U A S$-CD8::GFP larvae in either wild-type or exo70 ${ }^{K G 08057 / M B 04553}$ mutant background were fed with RU486 $20 \mathrm{~h}$ before reaching the wandering third instar larval stage. Immunohistochemistry was performed in the absence of Triton X-100. Comparison of intracellular $(\boldsymbol{B}, \boldsymbol{F})$ and surface $(\boldsymbol{C}, \boldsymbol{G})$ CD8::GFP at the boutons of control $(\boldsymbol{B}-\boldsymbol{E})$ and ex070 ${ }^{K G 08051 / M B 04553}$ mutant $(\boldsymbol{F}-\boldsymbol{I})$. Scale bar, $8 \mu \mathrm{m}$. J, Quantification of corrected fluorescence intensity of $\boldsymbol{B}-\boldsymbol{I}$. $n$ indicates the number of analyzed NMJs. All experiments were performed three times independently. Statistical analysis was performed using ANOVA. Error bars indicate SEM. ${ }^{* *} p<0.01$.

extensions that precede Type II bouton formation (Koon et al., 2011). Natural synaptopods are found on unstimulated Type II arbors, but more synaptopods can be induced by increasing activity via a high $\mathrm{K}^{+}$depolarization spaced stimulation paradigm (Ataman et al., 2008; Koon et al., 2011). First, we performed immunohistochemistry to confirm the expression of Exo70 in Type II boutons (Figs. 6B, 7A). Next, to visualize the octopaminergic Type II NMJs, we crossed the tyraminergic/octopaminergic neuron driver Tdc2-Gal4 to UAS-CD8::GFP in both control and exo70 KG08051/MB04553 mutants, and then performed time-lapse live imaging on Type II arbors in opened living larval preparations. A robust increase of synaptopods was observed in the control larvae after $5 \times$ spaced $\mathrm{K}^{+}$stimulation (Fig. $\left.6 C, D, G\right)(1.12 \pm$ 0.21 in control sham stim vs $4.23 \pm 0.54$ in control $5 \times \mathrm{K}^{+}, p=$ $0.0000175)$. However, this increase was significantly impaired in exo70 ${ }^{K G 08051 / M B 04553}$ mutants (Fig. $\left.7 C-G\right)(4.23 \pm 0.54$ in control $5 \times \mathrm{K}^{+}$vs $0.58 \pm 0.41$ in exo70 $0^{K G 08051 / M B 04553} 5 \times \mathrm{K}^{+}, p=$

(Figure legend continued.) L, Quantification of $\boldsymbol{H}-\boldsymbol{K} . \boldsymbol{M}$, Western blot of p-JNK, JNK, p-c-Jun, and C-Jun. $\boldsymbol{N}$, Confocal micrograph of Type II NMJs on muscles 12 and 13 of segment A4, colabeled with anti-TBH (green) and anti-HRP (red). $\mathbf{O}, \boldsymbol{P}$, Confocal micrographs of Type I NMJs on muscles 6 and 7 of segment A3, colabeled with anti-TBH (green) and anti-HRP (red). N, O, C380 driver control. $\boldsymbol{P},\left(380>\right.$ UAS-hep $^{C A} . n$ indicates the number of analyzed NMJs. All experiments were performed three times independently. Statistical analysis was performed using ANOVA. Error bars indicate SEM. ${ }^{*} p<0.05,{ }^{* *} p<0.01,{ }^{* * *} p<0.001$
0.0000063). Our data suggest that Exo70 is necessary for the induction of synaptopods by activity.

Other components of the exocyst complex, such as Sec6, have been shown to be enriched in axon growth cones and filopodia (Hazuka et al., 1999). Thus, we wondered whether synaptopods would contain Exo70. We fixed the opened living larval preparations after the $5 \times$ spaced $\mathrm{K}^{+}$stimulation and immunostained for Exo70. However, in contrast to our expectation, Exo70 was not detected in the synaptopods. Nevertheless, Exo70 was observed at regions of Type II arbors where the synaptopods were sprouting (Fig. $7 H$ ). Overexpression of Exo70 has been shown to produce filopodia that are devoid of actin, suggesting that Exo70 can bend the plasma membrane independent of actin remodeling (Zhao et al., 2013). Hence, the localization of Exo70 at synaptopod sprouting regions may be responsible for creating membrane curvatures to facilitate arbor extension.

\section{Exo70 is necessary for neurite arborization and survival under mild thermal stress}

Because Exo70 is required for JNK signaling- and activityinduced synaptic growth, we sought to determine whether it is also required for temperature-induced synaptic growth. Elevated temperature induces NMJ outgrowth (Sigrist et al., 2003). Thus, we reared control animals and exo 70 mutants at either $25^{\circ} \mathrm{C}$ or $29^{\circ} \mathrm{C}$, and analyzed their increase of boutons in response to elevated temperature. The bouton numbers in control animals in- 

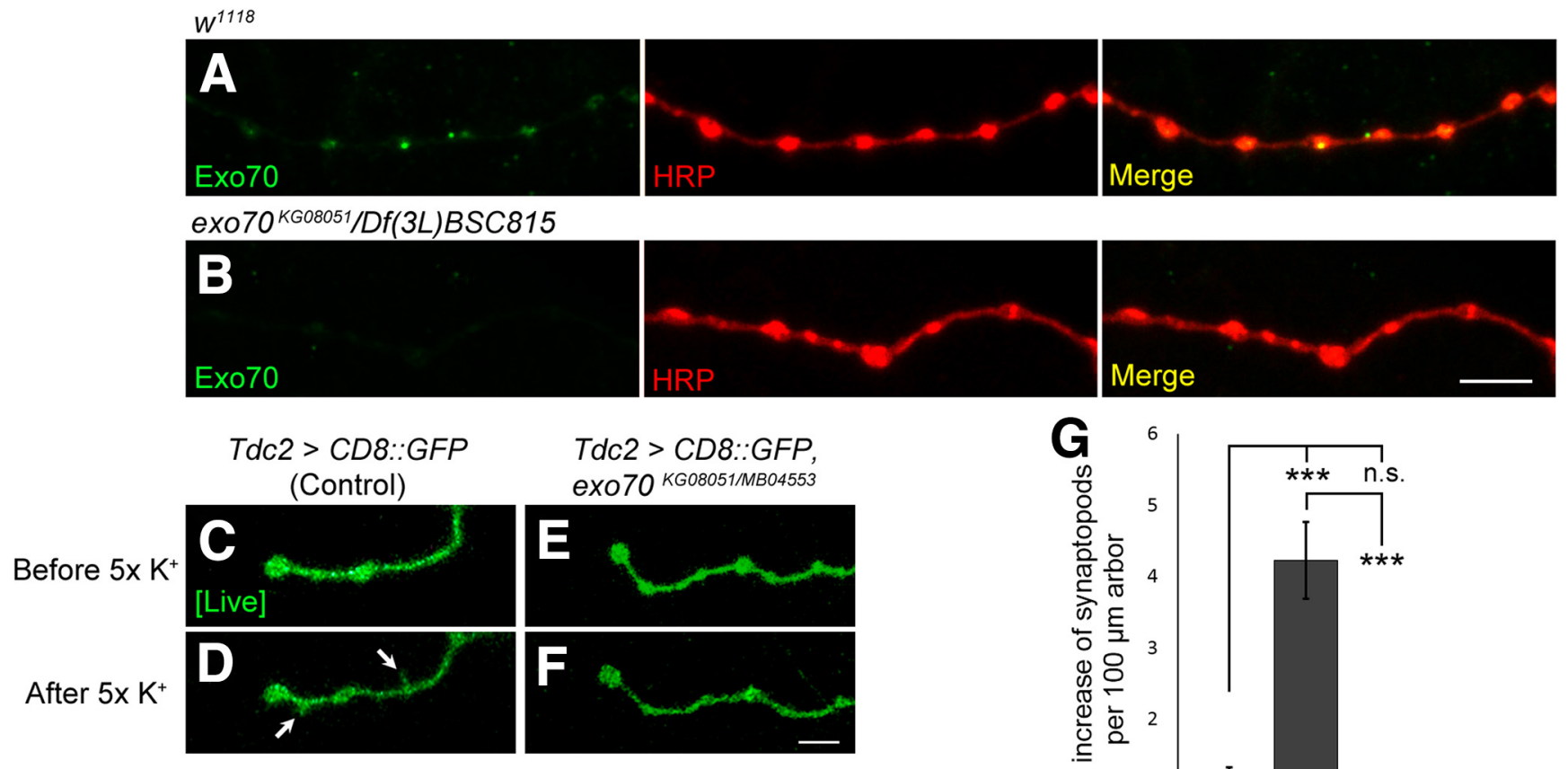

Tdc2: Type II motor neuron driver

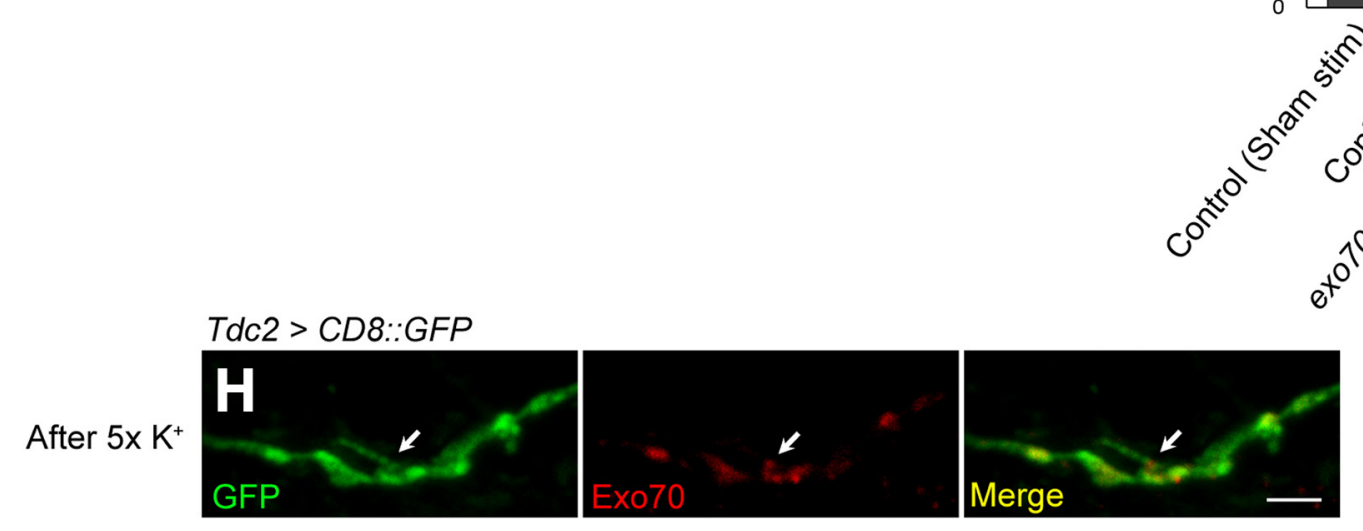

Figure 7. Exo70 is localized in Type II boutons and is required for activity-induced arbor extension. $\boldsymbol{A}, \boldsymbol{B}$, Confocal micrographs of Type II NMJs on segment A4, muscle 12. Type II boutons were colabeled with anti-Exo70 ${ }^{\text {Maguro }}$ (green) and anti-HRP (red). $\boldsymbol{A}, w^{1118}$. B, exo $70^{K G 08051} / D f(3 L) B S C 815$. Scale bar, $4 \mu \mathrm{m}$. C $\boldsymbol{F}$, Time-lapse live imaging of Type II arbors. C, D, Tdc2 > CD8::GFP. E, $\boldsymbol{F}$, TdC2 > CD8::GFP, ex070 ${ }^{K G 08057 / M B 04553}$. C, E, Before $5 \times$ spaced K ${ }^{+}$stimulation. D, F, After $5 \times$ spaced K ${ }^{+}$stimulation. Scale bar, $2.5 \mu$ m. G, Quantification of $\boldsymbol{C}-\boldsymbol{F}$. $\boldsymbol{H}$, Confocal micrograph of a synaptopods on Type Il arbor after stimulation, colabeled with anti-GFP (green) and anti-Exo $70^{\text {Maguro }}$ (red). Scale bar, $2.5 \mu \mathrm{m} . n$ indicates the number of analyzed Type II NMJs. All experiments were performed three times independently. Statistical analysis was performed using ANOVA. Error bars indicate SEM. ${ }^{* * *} p<0.001$.

creased in response to the elevated temperature $(82.2 \pm 2.7$ in $w^{1118}$ at $25^{\circ} \mathrm{C}$ vs $99.2 \pm 3.6$ in $w^{1118}$ at $29^{\circ} \mathrm{C}$ ), but this temperature-induced bouton increase was abolished in exo70 mutants (Fig. 8A).

Remarkably, when we reared these animals to adult stage at $29^{\circ} \mathrm{C}$, the exo70 mutants exhibited lethality (Fig. $8 B$ ). At $25^{\circ} \mathrm{C}$, both control animals and exo70 mutants showed nearly $0 \%$ lethality. However, at $29^{\circ} \mathrm{C}$, in which the animals experienced mild thermal stress, exo70 mutants exhibited significant pharate adult lethality $\left(\right.$ At $29^{\circ} \mathrm{C}, 0.00 \pm 0.0$ in $w^{1118}$ vs $98.4 \pm$ 1.1 in exo70 ${ }^{K G 08051 / K G 08051}, p=0.00000006 ; 0.00 \pm 0.0$ in $w^{1118}$ vs $96.7 \pm 1.6$ in exo $70^{K G 08051} / D f(3 L) B S C 815, p=0.00000030$; $0.00 \pm 0.0$ in $w^{1118}$ vs $73.1 \pm 7.8$ in exo70 1 MB04553/ $D f(3 L) B S C 815, p=0.00046695 ; 0.00 \pm 0.0$ in $w^{1118}$ vs $65.8 \pm$ 6.5 in exo70 $\left.{ }^{K G 08051 / M B 04553}, p=0.00035240\right)$. All the escapers that eclosed from the pupae died within $1 \mathrm{~h}$ of eclosion. We noticed no difference in the lethality rates of exo70 KG08051/KG08051 and exo $70^{K G 08051} / \mathrm{Df}(3 \mathrm{~L}) \mathrm{BSC} 815$ at $29^{\circ} \mathrm{C}$. By contrast, the lethality rates of exo70 $0^{M B 04553} / D f(3 L) B S C 815$ and exo70 $0^{K G 08051 / M B 04553}$ were less severe than those of exo70 KG08051/KG08051 and exo70 ${ }^{K G 08051} /$ $D f(3 L) B S C 815\left(96.7 \pm 1.6\right.$ in exo70 $0^{K G 08051} / D f(3 L) B S C 815$ vs $73.1 \pm$ 7.8 in exo70 ${ }^{M B 04553} / D f(3 L) B S C 815, p=0.004967 ; 96.7 \pm 1.6$ in

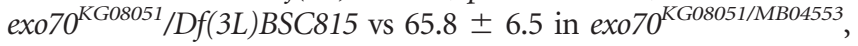
$p=0.000853)$. This can be explained by the hypomorphic nature of exo70 $\mathrm{MB04553}$.

To better understand the conditions of these mutants, we overexpressed UAS-CD8::GFP using Tdc2-GAL4 to visualize their tyraminergic/octopaminergic neurons. Although these neurons are not essential for viability, they allow us to monitor neurite arborization in the CNS. We reared the animals at either $25^{\circ} \mathrm{C}$ or $29^{\circ} \mathrm{C}$, and dissected the CNS of the freshly eclosed control animals and exo70 mutants (escapers at $29^{\circ} \mathrm{C}$ ). At $25^{\circ} \mathrm{C}$, no apparent defects were observed in the CNS of control animals and exo70 mutants (Fig. $8 C-F$ ). By contrast, at $29^{\circ} \mathrm{C}$, we found that the tyraminergic/octopaminergic neurons of the exo70 mutants had severe neurite arborization defects (Fig. $8 G-J$ ). These results 
A
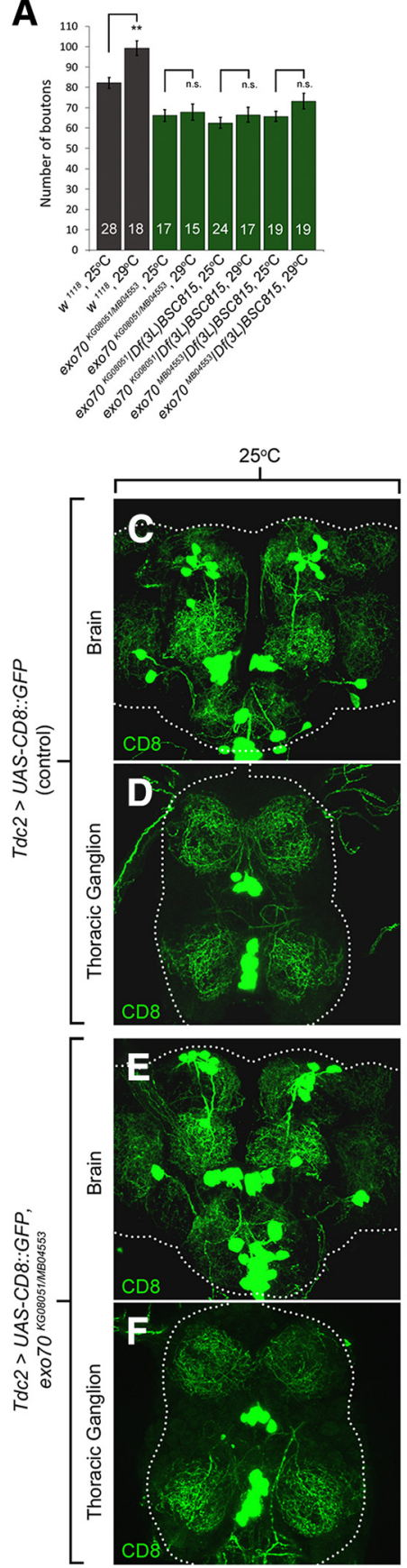

B
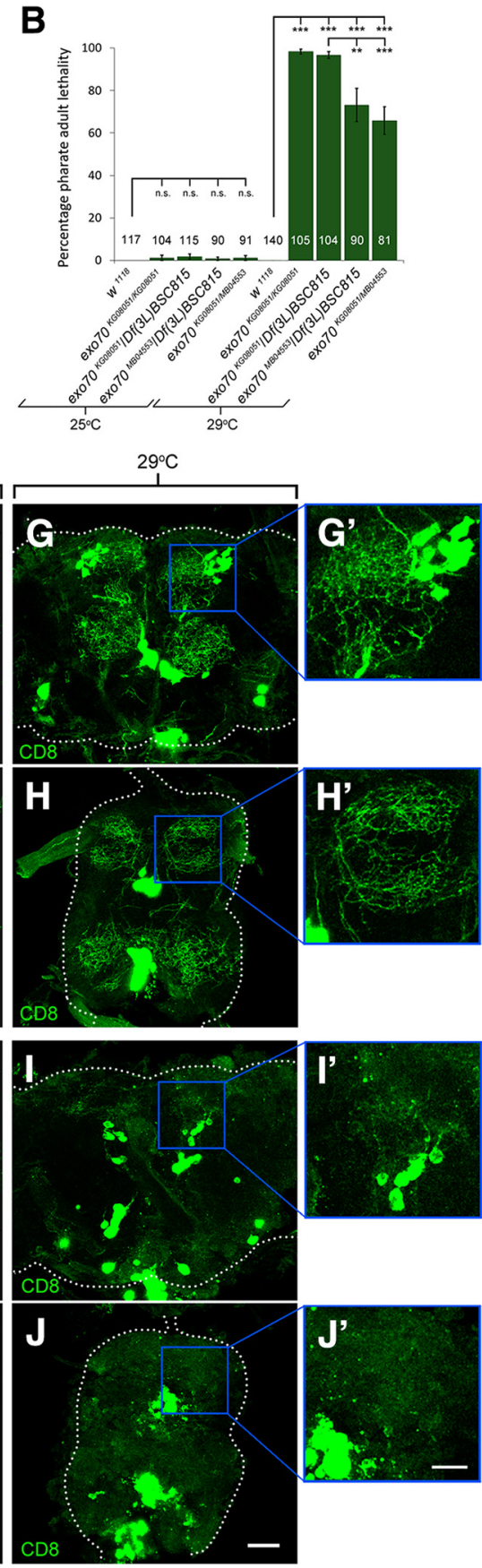
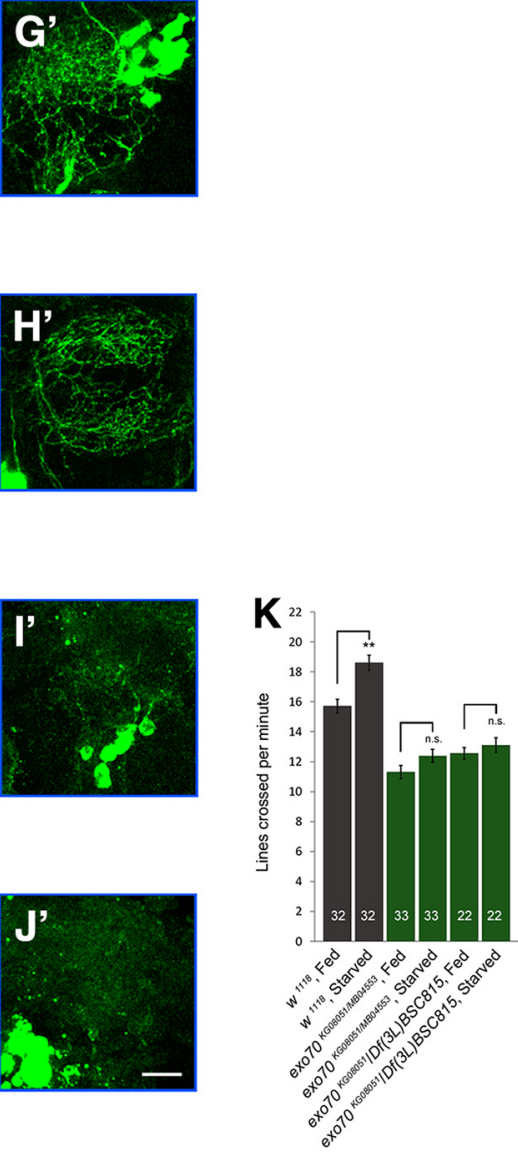

Figure 8. exo70 mutants exhibit neurite arborization and pharate adult lethality under thermal stress. A, Quantification of synaptic boutons on muscles 6 and 7 of segment A3. B, Quantification of pharate adult lethality (percentage). $\mathbf{C}-\mathbf{J}$, Confocal micrographs of the brains and thoracic ganglia of freshly eclosed adults reared at $25^{\circ} \mathrm{C}(\boldsymbol{C}-\boldsymbol{F})$ or $29^{\circ} \mathrm{C}(\boldsymbol{G}-\boldsymbol{J})$. $\mathbf{C}, \mathbf{D}, \mathbf{G}, \boldsymbol{H}$, The adult brain and thoracic ganglion of Tdc2 $>$ UAS-CD8::GFP controls. $\boldsymbol{G}^{\prime}, \boldsymbol{H}^{\prime}$, High magnification of $\mathbf{G}$ and $\boldsymbol{H}$, respectively. $\boldsymbol{E}, \boldsymbol{F}, \boldsymbol{I}, \boldsymbol{J}$, The adult brain and thoracic ganglion of Tdc2 $>$ UAS-CD8::GFP, ex070 ${ }^{K G 08057 / M B 04553}$. $I^{\prime}, J^{\prime}$, Higher magnification of $\boldsymbol{I}$ and $\boldsymbol{J}$, respectively. Scale bars: $\boldsymbol{C}-\boldsymbol{J}, 30 \mu \mathrm{m} ; \boldsymbol{G}^{\prime}, \boldsymbol{H}^{\prime}, \boldsymbol{I}^{\prime}, \boldsymbol{J}^{\prime}, 15 \mu \mathrm{m} . n$ indicates the number of analyzed animals. All experiments were performed three times independently. Statistical analysis was performed using ANOVA. Error bars indicate SEM.

indicated that exo70 mutants are unable to cope with thermal stress.

To investigate whether exo70 mutants are defective in adapting to other types of environmental stress, we conducted a starvation assay (Koon et al., 2011). We first performed the larval crawling assay on control animals and exo70 mutants to measure their locomotor activities. The same animals were then starved for $2 \mathrm{~h}$, and their locomotor activities were measured again. In response to starvation, control larvae significantly increased in locomotor activity (Fig. $8 \mathrm{~K}$ ). By contrast, exo70 mutants failed to increase in locomotor activity under the starved condition (Fig. $8 \mathrm{~K})$. Thus, exo70 mutants are defective in their behavioral response to starvation stress.

\section{Discussion}

In this study, we characterized two Drosophila mutant alleles of exo70 (Fig. 1). We demonstrated that presynaptic Exo70 is required for normal synaptic growth at the NMJ during development (Fig. 2). Presynaptic Exo70 was found to be localized in synaptic boutons, juxtaposed to active zones (Figs. 3, 4E,F). 
exo70 mutants had decreased glutamate receptor density and mEPSP amplitude (Fig. 4). We further observed a genetic interaction between exo70 and ralA in regulating synaptic growth (Fig. $5 B-F)$, but this regulation is independent of the JNK signaling pathway (Fig. 5H-M). exo70 mutants display defects in the delivery of integral membrane proteins from cell body to the synapses, which suggests a potential defect in the fusion of secretory vesicles to the plasma membrane at the terminals, and a subsequent deficit of membrane delivery to the terminals for the formation of new boutons (Fig. 6). In addition to the glutamatergic Type I motor neurons, Exo70 was also found in the octopaminergic Type II motor neurons at the NMJ (Fig. 7A,B). Furthermore, Exo70 was detected at regions where membrane extensions (synaptopods) are forming, and was required for activitydependent arbor extension (Fig. $7 \mathrm{C}-\mathrm{H}$ ). Consistent with these findings, both JNK signaling-induced synaptic growth (Fig. $5 H-L$ ) and elevated temperature-induced synaptic growth (Fig. $8 A$ ) at the NMJ were disrupted in exo70 mutants. Strikingly, the exposure to mild thermal stress resulted in severe neurite arborization defects and pharate adult lethality in the exo70 mutants (Fig. 8B-J). Last but not least, exo70 mutants are also defective in their starvation stress-induced locomotor behavior (Fig. $8 \mathrm{~K}$ ), underpinning the importance of Exo70 in the adaptation to different types of environmental stress.

The exocyst complex is widely known for mediating postGolgi secretory vesicle fusion during exocytosis (Novick et al., 1980; TerBush et al., 1996). However, the exocyst is not involved in the release of synaptic vesicles, as mutants in exocyst subunits, such as the sec5 mutants, have relatively normal evoked responses (Murthy et al., 2003). Here, we found that exo70 mutants exhibit significantly lower mEPSP amplitude (Fig. 4G,I), which might be caused by their lower density of glutamate receptors (Fig. 4A-C). Consistent with past studies, our results show that EPSP amplitude is intact in exo70 KG08051/MBO4553 mutants and only slightly reduced in exo70 ${ }^{K G 08051} / D f(3 L) B S C 815$ mutants (Fig. 4K). The reason why the EPSP amplitudes are relatively normal in exo70 mutants is likely because of a homeostatic increase in presynaptic release to compensate for the loss of glutamate receptors (Yeates et al., 2017). This was reflected by the overall increase of QC in exo70 mutants (Fig. $4 L$ ).

The small GTPase RalA is known to regulate the JNK signaling pathway under oxidative stress (van den Berg et al., 2013). In Drosophila, it has been shown that RalA functions together with the exocyst complex to negatively regulate JNK-induced apoptosis (Balakireva et al., 2006). At the Drosophila NMJ, it has been reported that postsynaptic RalA is required for activitydependent growth of the subsynaptic reticulum via its interaction with the exocyst (Teodoro et al., 2013). In the presynaptic motor neuron, oxidative stress induces synaptic growth at the NMJ via the JNK pathway (Milton et al., 2011). However, at the NMJ, whether RalA is involved in presynaptic outgrowth is unclear. Our data demonstrated that RalA functions together with Exo70 to positively regulate presynaptic outgrowth (Fig. $5 A-F$ ). But the regulation of synaptic growth by Exo70 does not involve the JNK pathway (Fig. $5 H-M$ ). Thus, the negative regulation of JNK by RalA is not involved either. In contrast, synaptic growth induced by the increase of JNK signaling is dependent on functional Exo70 (Fig. $5 H-L$ ).

Because the regulation of synaptic growth by Exo70 and RalA does not involve the JNK pathway, it could involve the exocystRalA-mediated transport and exocytosis of secretory vesicles via GTP binding (Sugihara et al., 2002; Wang et al., 2004), which is a crucial cellular process in delivering newly synthesized mem- branes to sites of neuronal outgrowth. Using an existing assay to examine the transport of CD8::GFP to the cell surface (Murthy et al., 2003), we found that exo70 mutations do not impair the transport of integral membrane proteins from the soma to synaptic boutons, but they disrupt the delivery of the proteins to the cell surface (Fig. 6). This result was different from what was observed in sec 5 mutants, in which even the transport of the proteins to the terminals was perturbed (Murthy et al., 2003). These findings are consistent with the notion that Sec5 is required for the primary assembly of the exocyst, whereas Exo70 is specifically involved in the fusion of vesicles to the plasma membrane via its interaction with the plasma membrane. Because secretory vesicle fusion is a major source of phospholipids for membrane extension, this may explain why exo70 mutations blocked all three kinds of induced synaptic growth (JNK, activity, and temperature) that were investigated in this study.

In addition to the glutamatergic Type I motor neurons, Exo70 was also present in octopaminergic Type II motor neurons at the NMJ (Fig. $7 A, B$ ). Although the exocyst complex is unlikely to be involved in the release of synaptic vesicles in Type I bouton, it may participate in the release of octopamine in Type II boutons. Octopamine is a potent neuromodulator of plasticity in Drosophila and plays a role in the synaptic growth of both Type I and Type II motor neurons (Koon et al., 2011). In particular, this biogenic amine is specifically involved in Type II arborization during starvation stress (Koon et al., 2011). But whether the exocyst is present and involved in the outgrowth of octopaminergic neurons was unclear. Our data clearly demonstrated Exo70's presence and its involvement in the activity-induced outgrowth of octopaminergic neurons (Fig. $7 C-G$ ). Considering that exo70 mutations restrict vesicle fusion at synaptic terminals, they may block the release of octopamine, which in turn impedes neurite arborization under environmental stress. This is in line with our observation that exo 70 mutant larvae are defective in starvation-induced locomotor activity increase (Fig. $8 \mathrm{~K}$ ), which resembles the $t b h$ mutants lacking octopamine (Koon et al., 2011).

Although Exo70 can mediate a wide range of cellular processes (Gerges et al., 2006; He et al., 2007; Dupraz et al., 2009; Zhao et al., 2013; Ma et al., 2016), exo70 mutants or knock-outs have not been reported to display any phenotype as severe as lethality. We are first to report a lethality phenotype in exo70 mutants under mild thermal stress (Fig. 8B), underpinning its importance in the organism's adaptation to environmental changes. This is vastly different from other mutants of exocyst subunits, such as sec 5 and $\sec 6$, which are both larval lethal due to growth arrest at $96 \mathrm{~h}$ after eye laying (AEL) and have much more severe NMJ phenotypes at $25^{\circ} \mathrm{C}$ (Murthy et al., 2003, 2005). The differences in the severity of lethality of these exocyst subunit mutants may be explained by the differences in the functions of individual subunits. As mentioned earlier, Sec5 is likely required for the primary assembly of the exocyst complex, whereas Exo70 is likely only required for regulating the fusion of vesicles with the plasma membrane. Seeing how the subcellular localization of Exo70 to be specifically juxtaposed to active zones (Fig. 4E,F), Exo70 may also be involved in directing the vesicle to dock at appropriate locations on the plasma membrane. Trafficking of vesicles is severely hampered in sec5 mutants (Murthy et al., 2003) but relatively normal in exo70 mutants (Fig. 6). It is possible that exocytosis is not completely abolished in exo70 mutants. Although inefficient, given sufficient time, essential proteins may still be delivered to the plasma membrane. However, environmental stress, such as elevated temperature, may accelerate the need for exocytosis, especially during metamorphosis, which requires the release of 
neuropeptides and the drastic remodeling of the nervous system. A vast amount of induced synaptic growth takes place during this period. Failure of proper docking of vesicles at specific locations may be one of the many causes for the lethality exhibited by exo70 mutants, as newly synthesized membranes are unable to effectively reach their designated regions for neurite extension or cell growth. On the other hand, the infertility phenotype of exo70 mutants may be similar to those of sec5 and sec 6 germline clones (Murthy et al., 2003, 2005). In the ovaries of sec5 and sec6 germline clones, the egg chambers have the phenotypes of ring canals clumping and loss of cell membrane between cells (Murthy et al., 2005). Future analysis of the ovaries of exo70 mutants will confirm whether exo70 mutants have similar cellular defects, and will provide us with more insights regarding the role of Exo70 in fertility.

In conclusion, this study presents Exo70 as an important regulator of induced synaptic growth and neurite extension by mediating secretory vesicle fusion at synaptic terminals. Exo70 is also crucial for survival under thermal stress.

\section{References}

Ataman B, Ashley J, Gorczyca M, Ramachandran P, Fouquet W, Sigrist SJ, Budnik V (2008) Rapid activity-dependent modifications in synaptic structure and function require bidirectional wnt signaling. Neuron 57: 705-718. CrossRef Medline

Bai Y, Nguyen L, Song Z, Peng S, Lee J, Zheng N, Kapoor I, Hagler LD, Cai K, Cheng J, Chan HY, Zimmerman SC (2016) Integrating display and delivery functionality with a cell penetrating peptide mimic as a scaffold for intracellular multivalent multitargeting. J Am Chem Soc 138:9498-9507. CrossRef Medline

Balakireva M, Rossé C, Langevin J, Chien YC, Gho M, Gonzy-Treboul G, Voegeling-Lemaire S, Aresta S, Lepesant JA, Bellaiche Y, White M, Camonis J (2006) The Ral/exocyst effector complex counters c-jun $\mathrm{N}$-terminal kinase-dependent apoptosis in Drosophila melanogaster. Mol Cell Biol 26:8953-8963. CrossRef Medline

Biondini M, Sadou-Dubourgnoux A, Paul-Gilloteaux P, Zago G, Arslanhan MD, Waharte F, Formstecher E, Hertzog M, Yu J, Guerois R, Gautreau A, Scita G, Camonis J, Parrini MC (2016) Direct interaction between exocyst and wave complexes promotes cell protrusions and motility. J Cell Sci 129:3756-3769. CrossRef Medline

Budnik V, Koh YH, Guan B, Hartmann B, Hough C, Woods D, Gorczyca M (1996) Regulation of synapse structure and function by the Drosophila tumor suppressor gene dlg. Neuron 17:627-640. CrossRef Medline

Cho B, Fischer JA (2011) Ral GTPase promotes asymmetric notch activation in the Drosophila eye in response to Frizzled/PCP signaling by repressing ligand-independent receptor activation. Development 138: 1349-1359. CrossRef Medline

Ding Y, Robinson DG, Jiang L (2014a) Unconventional protein secretion (UPS) pathways in plants. Curr Opin Cell Biol 29:107-115. CrossRef Medline

Ding Y, Wang J, Chun Lai JH, Ling Chan VH, Wang X, Cai Y, Tan X, Bao Y, Xia J, Robinson DG, Jiang L (2014b) Exo70E2 is essential for exocyst subunit recruitment and EXPO formation in both plants and animals. Mol Biol Cell 25:412-426. CrossRef Medline

Dubuque SH, Schachtner J, Nighorn AJ, Menon KP, Zinn K, Tolbert LP (2001) Immunolocalization of synaptotagmin for the study of synapses in the developing antennal lobe of manduca sexta. J Comp Neurol 441: 277-287. CrossRef Medline

Dupraz S, Grassi D, Bernis ME, Sosa L, Bisbal M, Gastaldi L, Jausoro I, Cáceres A, Pfenninger KH, Quiroga S (2009) The TC10-Exo70 complex is essential for membrane expansion and axonal specification in developing neurons. J Neurosci 29:13292-13301. CrossRef Medline

Etter PD, Narayanan R, Navratilova Z, Patel C, Bohmann D, Jasper H, Ramaswami M (2005) Synaptic and genomic responses to JNK and AP-1 signaling in Drosophila neurons. BMC Neurosci 6:39. CrossRef Medline

Eun SH, Lea K, Overstreet E, Stevens S, Lee JH, Fischer JA (2007) Identification of genes that interact with Drosophila liquid facets. Genetics 175: 1163-1174. CrossRef Medline

Fujita A, Koinuma S, Yasuda S, Nagai H, Kamiguchi H, Wada N, Nakamura
T (2013) GTP hydrolysis of TC10 promotes neurite outgrowth through exocytic fusion of Rab11- and L1-containing vesicles by releasing exocyst component Exo70. PLoS One 8:e79689. CrossRef Medline

Fujita SC, Zipursky SL, Benzer S, Ferrús A, Shotwell SL (1982) Monoclonal antibodies against the Drosophila nervous system. Proc Natl Acad Sci U S A 79:7929-7933. CrossRef Medline

Gerges NZ, Backos DS, Rupasinghe CN, Spaller MR, Esteban JA (2006) Dual role of the exocyst in AMPA receptor targeting and insertion into the postsynaptic membrane. EMBO J 25:1623-1634. CrossRef Medline

Hála M, Cole R, Synek L, Drdová E, Pecenková T, Nordheim A, Lamkemeyer T, Madlung J, Hochholdinger F, Fowler JE, Zárský V (2008) An exocyst complex functions in plant cell growth in arabidopsis and tobacco. Plant Cell 20:1330-1345. CrossRef Medline

Harris D, Orme C, Kramer J, Namba L, Champion M, Palladino MJ, Natzle J, Hawley RS (2003) A deficiency screen of the major autosomes identifies a gene (matrimony) that is haplo-insufficient for achiasmate segregation in Drosophila oocytes. Genetics 165:637-652. Medline

Hazuka CD, Foletti DL, Hsu SC, Kee Y, Hopf FW, Scheller RH (1999) The sec6/8 complex is located at neurite outgrowth and axonal synapseassembly domains. J Neurosci 19:1324-1334. CrossRef Medline

He B, Xi F, Zhang X, Zhang J, Guo W (2007) Exo70 interacts with phospholipids and mediates the targeting of the exocyst to the plasma membrane. EMBO J 26:4053-4065. CrossRef Medline

Hertzog M, Monteiro P, Le Dez G, Chavrier P (2012) Exo70 subunit of the exocyst complex is involved in adhesion-dependent trafficking of caveolin-1. PLoS One 7:e52627. CrossRef Medline

Hoang B, Chiba A (2001) Single-cell analysis of Drosophila larval neuromuscular synapses. Dev Biol 229:55-70. CrossRef Medline

Hong W, Lev S (2014) Tethering the assembly of SNARE complexes. Trends Cell Biol 24:35-43. CrossRef Medline

Inoue M, Chang L, Hwang J, Chiang SH, Saltiel AR (2003) The exocyst complex is required for targeting of Glut 4 to the plasma membrane by insulin. Nature 422:629-633. CrossRef Medline

Koon AC, Ashley J, Barria R, DasGupta S, Brain R, Waddell S, Alkema MJ, Budnik V (2011) Autoregulatory and paracrine control of synaptic and behavioral plasticity by octopaminergic signaling. Nat Neurosci 14:190199. CrossRef Medline

Li L, Ng NK, Koon AC, Chan HY (2017) Expanded polyalanine tracts function as nuclear export signals and promote protein mislocalization via eEF1A1 factor. J Biol Chem 292:5784-5800. CrossRef Medline

Liebl FL, Chen K, Karr J, Sheng Q, Featherstone DE (2005) Increased synaptic microtubules and altered synapse development in Drosophila sec8 mutants. BMC Biol 3:27. CrossRef Medline

Lobo GP, Fulmer D, Guo L, Zuo X, Dang Y, Kim SH, Su Y, George K, Obert E, Fogelgren B, Nihalani D, Norris RA, Rohrer B, Lipschutz JH (2017) The exocyst is required for photoreceptor ciliogenesis and retinal development. J Biol Chem 292:14814-14826. CrossRef Medline

Ma W, Wang Y, Yao X, Xu Z, An L, Yin M (2016) The role of Exo70 in vascular smooth muscle cell migration. Cell Mol Biol Lett 21:20. CrossRef Medline

Milton VJ, Jarrett HE, Gowers K, Chalak S, Briggs L, Robinson IM, Sweeney ST (2011) Oxidative stress induces overgrowth of the Drosophila neuromuscular junction. Proc Natl Acad Sci U S A 108:17521-17526. CrossRef Medline

Monastirioti M, Gorczyca M, Rapus J, Eckert M, White K, Budnik V (1995) Octopamine immunoreactivity in the fruit fly Drosophila melanogaster. J Comp Neurol 356:275-287. CrossRef Medline

Murthy M, Garza D, Scheller RH, Schwarz TL (2003) Mutations in the exocyst component Sec5 disrupt neuronal membrane traffic, but neurotransmitter release persists. Neuron 37:433-447. CrossRef Medline

Murthy M, Ranjan R, Denef N, Higashi ME, Schupbach T, Schwarz TL (2005) Sec6 mutations and the Drosophila exocyst complex. J Cell Sci 118:1139-1150. CrossRef Medline

Novick P, Field C, Schekman R (1980) Identification of 23 complementation groups required for post-translational events in the yeast secretory pathway. Cell 21:205-215. CrossRef Medline

Osterwalder T, Yoon KS, White BH, Keshishian H (2001) A conditional tissue-specific transgene expression system using inducible GAL4. Proc Natl Acad Sci U S A 98:12596-12601. CrossRef Medline

Parnas D, Haghighi AP, Fetter RD, Kim SW, Goodman CS (2001) Regulation of postsynaptic structure and protein localization by the rho-type 
guanine nucleotide exchange factor dPix. Neuron 32:415-424. CrossRef Medline

Petersen SA, Fetter RD, Noordermeer JN, Goodman CS, DiAntonio A (1997) Genetic analysis of glutamate receptors in Drosophila reveals a retrograde signal regulating presynaptic transmitter release. Neuron 19: 1237-1248. CrossRef Medline

Sanyal S, Sandstrom DJ, Hoeffer CA, Ramaswami M (2002) AP-1 functions upstream of CREB to control synaptic plasticity in Drosophila. Nature 416:870-874. CrossRef Medline

Shen W, Ganetzky B (2009) Autophagy promotes synapse development in Drosophila. J Cell Biol 187:71-79. CrossRef Medline

Sigrist SJ, Reiff DF, Thiel PR, Steinert JR, Schuster CM (2003) Experiencedependent strengthening of Drosophila neuromuscular junctions. J Neurosci 23:6546-6556. CrossRef Medline

Sugihara K, Asano S, Tanaka K, Iwamatsu A, Okawa K, Ohta Y (2002) The exocyst complex binds the small GTPase RalA to mediate filopodia formation. Nat Cell Biol 4:73-78. CrossRef Medline

Teodoro RO, Pekkurnaz G, Nasser A, Higashi-Kovtun ME, Balakireva M, McLachlan IG, Camonis J, Schwarz TL (2013) Ral mediates activitydependent growth of postsynaptic membranes via recruitment of the exocyst. EMBO J 32:2039-2055. CrossRef Medline

TerBush DR, Maurice T, Roth D, Novick P (1996) The exocyst is a multiprotein complex required for exocytosis in saccharomyces cerevisiae. EMBO J 15:6483-6494. Medline

van den Berg MC, van Gogh IJ, Smits AM, van Triest M, Dansen TB, Visscher M, Polderman PE, Vliem MJ, Rehmann H, Burgering BM (2013) The small GTPase RALA controls c-jun N-terminal kinase-mediated FOXO activation by regulation of a JIP1 scaffold complex. J Biol Chem 288: 21729-21741. CrossRef Medline

Wagh DA, Rasse TM, Asan E, Hofbauer A, Schwenkert I, Dürrbeck H, Buchner S, Dabauvalle MC, Schmidt M, Qin G, Wichmann C, Kittel R, Sigrist SJ, Buchner E (2006) Bruchpilot, a protein with homology to ELKS/
CAST, is required for structural integrity and function of synaptic active zones in Drosophila. Neuron 49:833-844. CrossRef Medline

Wang J, Ding Y, Wang J, Hillmer S, Miao Y, Lo SW, Wang X, Robinson DG, Jiang L (2010) EXPO, an exocyst-positive organelle distinct from multivesicular endosomes and autophagosomes, mediates cytosol to cell wall exocytosis in arabidopsis and tobacco cells. Plant Cell 22:4009-4030. CrossRef Medline

Wang L, Li G, Sugita S (2004) RalA-exocyst interaction mediates GTPdependent exocytosis. J Biol Chem 279:19875-19881. CrossRef Medline

Xiao L, Zheng K, Lv X, Hou J, Xu L, Zhao Y, Song F, Fan Y, Cao H, Zhang W, Hong X, Zhan YY, Hu T (2017) Exo70 is an independent prognostic factor in colon cancer. Sci Rep 7:5039. CrossRef Medline

Yeates CJ, Zwiefelhofer DJ, Frank CA (2017) The maintenance of synaptic homeostasis at the Drosophila neuromuscular junction is reversible and sensitive to high temperature. eNeuro 4:ENEUOR.0220-17.2017. CrossRef Medline

Zhang C, Brown MQ, van de Ven W, Zhang ZM, Wu B, Young MC, Synek L, Borchardt D, Harrison R, Pan S, Luo N, Huang YM, Ghang YJ, Ung N, Li R, Isley J, Morikis D, Song J, Guo W, Hooley RJ, et al. (2016) Endosidin2 targets conserved exocyst complex subunit EXO70 to inhibit exocytosis. Proc Natl Acad Sci U S A 113:E41-E50. CrossRef Medline

Zhao Y, Liu J, Yang C, Capraro BR, Baumgart T, Bradley RP, Ramakrishnan N, Xu X, Radhakrishnan R, Svitkina T, Guo W (2013) Exo70 generates membrane curvature for morphogenesis and cell migration. Dev Cell 26:266-278. CrossRef Medline

Zhao Y, Hou J, Mi P, Mao L, Xu L, Zhang Y, Xiao L, Cao H, Zhang W, Zhang B, Song G, Hu T, Zhan YY (2016) Exo70 is transcriptionally upregulated by hepatic nuclear factor 4alpha and contributes to cell cycle control in hepatoma cells. Oncotarget 7:9150-9162. CrossRef Medline

Zuo X, Zhang J, Zhang Y, Hsu SC, Zhou D, Guo W (2006) Exo70 interacts with the Arp2/3 complex and regulates cell migration. Nat Cell Biol 8:1383-1388. CrossRef Medline 